\title{
High-redshift cosmography: auxiliary variables versus Padé polynomials
}

\author{
S. Capozziello,,$^{1,2,3 \star}$ R. D’Agostino ${ }^{1,2} \dagger$ and O. Luongo ${ }^{4} \ddagger$ \\ 1 Dipartimento di Fisica "E. Pancini", Università di Napoli "Federico II", 80126 Napoli, Italy. \\ 2 INFN, Sezione di Napoli, Complesso Universitario di Monte S. Angelo, Via Cintia Edificio 6, 80126 Napoli, Italy. \\ ${ }^{3}$ Laboratory for Theoretical Cosmology, Tomsk State University of Control Systems and Radioelectronics (TUSUR), 634050 Tomsk, Russia, \\ 4 INFN, Laboratori Nazionali di Frascati, 00044 Frascati, Via Enrico Fermi, Italy.
}

Accepted XXX. Received YYY; in original form ZZZ

\begin{abstract}
Cosmography becomes non-predictive when cosmic data span beyond the red shift limit $z \simeq 1$. This leads to a strong convergence issue that jeopardizes its viability. In this work, we critically compare the two main solutions of the convergence problem, i.e. the $y$-parametrizations of the redshift and the alternatives to Taylor expansions based on Padé series. In particular, among several possibilities, we consider two widely adopted parametrizations, namely $y_{1}=1-a$ and $y_{2}=\arctan \left(a^{-1}-1\right)$, being $a$ the scale factor of the Universe. We find that the $y_{2}$-parametrization performs relatively better than the $y_{1}$-parametrization over the whole redshift domain. Even though $y_{2}$ overcomes the issues of $y_{1}$, we get that the most viable approximations of the luminosity distance $d_{L}(z)$ are given in terms of Padé approximations. In order to check this result by means of cosmic data, we analyze the Padé approximations up to the fifth order, and compare these series with the corresponding $y$-variables of the same orders. We investigate two distinct domains involving Monte Carlo analysis on the Pantheon Superovae Ia data, $H(z)$ and shift parameter measurements. We conclude that the $(2,1)$ Padé approximation is statistically the optimal approach to explain low and high-redshift data, together with the fifth-order $y_{2}$-parametrization. At high redshifts, the $(3,2)$ Padé approximation cannot be fully excluded, while the $(2,2)$ Padé one is essentially ruled out.
\end{abstract}

Key words: cosmological parameters - dark energy - observations

\section{INTRODUCTION}

The Universe dynamics is currently undergoing an accelerated phase driven by a dark energy component, which can assume the form of a cosmological constant $(\Lambda)$ originated by the quantum fluctuations of early vacuum (Sahni \& Starobinsky 2000; Carroll 2001; Peebles \& Ratra 2003; Copeland, Sami \& Tsujikawa 2006; Dunsby, Luongo \& Reverberi 2016; Luongo \& Muccino 2018). A wide experimental evidence suggests the concordance flat $\Lambda$ CDM paradigm as the most successful model to explain early and late time dynamics. However, the recent developments of the Planck collaboration (Akrami et al. 2018) seems to indicate that corrections to Einstein's gravity can explain the cosmological inflation immediately after the Big Bang and confirm the so called Starobinsky model (Starobinsky 1979). Hence, possibility that a slightly evolving dark energy contribution can fuel the energy momentum-tensor, alleviating the problems related to the today tiny value ${ }^{1}$ of $\Lambda$, is still valid. Thus, distinguishing dark energy from $\Lambda$ and understanding whether the equation of state of the Universe is varying (Chevallier \& Polarski 2001; Linder 2003; Padmanabhan 2010; Tsujikawa 2010; Aviles \& Cervantes-Cota 2011; Clifton et al. 2012; Bamba et al. 2012; Luongo \& Quevedo 2014a,b; Piedipalumbo et al. 2014) have widely prompted the search for model-independent treatments and null-diagnostics (Harrison 1976; Visser 2005; Uzan, Clarkson \& Ellis 2008; Clarkson, Bassett \& Lu 2008; Zunckel \& Clarkson 2008; Sahni, Shafieloo \& Starobinsky 2008; Nesseris \& Shafieloo 2010; Farooq \& Ratra 2013; Busti, Holanda \& Clarkson 2013; Aviles et al. 2014; Escamilla-Rivera \& Capozziello 2019; Escamilla-Rivera, Quintero \& Capozziello 2020;

1 This heals $d \mathrm{e}$ facto the thorny issue of fine-tuning plaguing the model (Weinberg 1989).

\footnotetext{
* capozzie@na.infn.it

† rocco.dagostino@na.infn.it

† orlando.luongo@lnf.infn.it
} 
Lobo, Mimoso \& Visser 2020). Model-independent techniques are constructed to give hints on the correct form of dark energy. However, the main issue is related to the origin of such a dark component which could come from some material field or be the cumulative effect of some modified gravity (Capozziello 2002; Nojiri \& Odintsov 2007; Amendola \& Tsujikawa 2015; Nojiri, Odintsov \& Oikonomou 2017).

Among the possible model-independent strategies, great emphasis has been given to the well-consolidated approach named cosmography (Visser 2015; Dunsby \& Luongo 2016; Capozziello, D'Agostino \& Luongo 2019). The advantage of cosmography consists in assuming only the homogeneity and isotropy of the Universe ${ }^{2}$, factorizing the scale factor around the present time. However, two main problems are associated to the use of the above expansion and analysis of the cosmic data. First, distinguishing the evolution of dark energy from $\Lambda$ requires highly refined limits over the derivatives of $a(t)$. So, one needs a wide number of data points to reduce cosmic systematics and to provide constraints over the cosmographic coefficients. In this sense, the lack of many and precise data limits the use of cosmography. A way of improving the quality of numerical fits may be using combined data sets or mock compilations based on the sensitivity and design of future surveys (D'Agostino \& Nunes 2019; Bonilla et al. 2020). The second problem, is based on the form of data. In fact, in order to determine possible departures from the standard cosmological model, one takes data which exceed the limit $t \simeq t_{0}$, with $t_{0}$ today epoch, or, alternatively speaking, the bound $z \simeq 0$. In other words, one faces a convergence problem caused by data that are far from the limits of the Taylor expansions, leading to a severe error propagation, which reduces the cosmographic predictions (Busti et al. 2015). To solve this problem, several approaches have been proposed so far in the literature. One of these relies on the use of auxiliary variables. The idea is to re-parametrize the redshift variable through functions of $z$, and expand in series of the cosmological observables. These functions should vanish at $z=0$ and converge to a finite number at $z \rightarrow \infty$. Another relevant possibility is to consider a smooth evolution of the involved observables by expanding them in terms of rational approximations. A feasible consequence of this scheme is the stability of these new expansions over a large redshift interval. Examples of this approach are the Padé and Chebyshev rational polynomials (Wei, Yan \& Zhou 2014; Gruber \& Luongo 2014; Capozziello, D'Agostino \& Luongo 2018a,b; Capozziello, Ruchika \& Sen 2019; Capozziello, D'Agostino \& Luongo

2019 ;

Benetti \& Capozziello 2019).

In this paper, we want to show how to significantly reduce the convergence problem, overcoming the weakness of cosmography by constructing the most suitable expansions to fix refined bounds over cosmographic coefficients. Thus, to handle the convergence issue, we first theoretically investigate the advantages of rational polynomials against auxiliary variables. In particular, we show that Padé polynomials

2 Cosmography assumes the validity of the cosmological principle and turns out to be purely model-independent in terms of derivatives of the scale factor. well adapt to match the cosmic dynamics at high redshifts. Based on this result which substantially agrees with previous ones in literature, we proceed to select the most suitable order of Padé expansion. To do so, we compare the two classes of approaches, i.e. the one making use of the auxiliary variables and the second using Padé series of different orders. We therefore propose a few conditions that every rational approximation should fulfill in order to be more predictive than a given parametrization. Moreover, we discuss that any new class of auxiliary variables, extending the role played by $y_{1}$ and $y_{2}$, turn out to be less predictive than Padé approximations. In particular, we split our discussion considering two regimes corresponding to low and high redshifts. In the low-redshift regime, we note that the results of the thirdorder $y_{1}$-variable are substantially different by $2 \sigma$ from the corresponding Taylor constraints, whereas the $(2,1)$ Padé polynomial turns out to be the best-performing third-order approximation. We compare $y_{2}$ with $y_{1}$ and we find that, at the fourth-order expansion, still the $y_{2}$-variable behaves better than the $y_{1}$-variable, while a persistent disfavour against the $(2,2)$ Padé polynomial is present, if compared to the $(2,1)$ Padé polynomial. At the fifth-order expansion, all the techniques are characterized by large uncertainties on the parameters beyond $s_{0}$, leaving open the possibility to use $(3,2)$ Padé polynomial, but disfavouring the corresponding $y$-variables. At high redshifts, we find that the most suitable approach is the $(2,1)$ Padé polynomial, showing, in all cases, a very strong evidence against the $y$-variables. However, for the sake of clearness, a relevant fact is offered by the $y_{2}$ variable which, at the fifth order, seems to better frame the cosmographic curves. In any case, we rule out the use of the $(2,2)$ Padé rational polynomial that does not represent a suitable approximation of $d_{L}(z)$. We statistically analyze all the predictions of the different approximations by means of Bayesian selection criteria. In view of our theoretical considerations and the statistical inference results, we conclude that cosmography built upon $(2,1)$ Padé polynomial remains the most suitable one at both low and highredshift domains. To quantitatively show this, we involve the most recent Supernova Ia data, the direct $H(z)$ measurements and the early time measurements of the cosmic microwave background (CMB) shift parameter.

The paper is structured as follows. After this introduction, we develop the main features of the cosmographic technique in Section 2. In particular, we discuss how to build up the cosmographic series and the basic demands of cosmography. We then face the convergence problem of the cosmographic series in Section 3, and we discuss the possible solutions to it. In particular, we introduce the concept of rational approximations and auxiliary variables, dealing with the concepts of Padé and $y$-cosmography respectively. We then show how to construct theoretically the most suitable approximation, by means of Padé polynomial first and then through the $y$-variables. In Section 4, we develop our numerical analyses by means of a hierarchy among coefficients. We handle different orders, starting from the third one, and then increasing it up to the fifth order. For each order, we analyze separately low and high-redshift data and we find constraints and convergence for each approximations. Hence, we compare our expectations with the numerical results, and the different orders by means of statistical criteria. Finally, the conclusions and perspectives of our work are reported in 
Section 5. In the Appendix, the cosmographic expansions, up to fifth order, are reported.

Throughout the paper, we use natural units with $c=1$.

\section{THE COSMOGRAPHIC APPROACH}

To describe the homogeneous and isotropic universe, we consider the Friedmann-Lemaître-Robertson-Walker (FLRW) line element, given by:

$d s^{2}=d t^{2}-a(t)^{2}\left[\frac{d r^{2}}{1-k r^{2}}+r^{2} d l^{2}\right]$,

where the present-day value of the scale factor is conventionally normalized to the unity (i.e. $a_{0}=1$ ), and $d l^{2} \equiv$ $\left(d \theta^{2}+\sin ^{2} \theta d \phi^{2}\right)$. Here, $k$ defines the spatial curvature. Dynamics of the universe is determined by solving the Friedmann equations:

$H^{2}=\frac{1}{3} \rho-\frac{k}{a^{2}}, \quad \dot{H}+H^{2}=-\frac{1}{6}(3 P+\rho)$,

where $H \equiv \dot{a} / a$ is the Hubble expansion rate; $\rho$ and $P$ are the energy density and pressure of the cosmic fluid, respectively. The cosmological dynamical system is completed considering the continuity equation $\dot{\rho}+3 H(\rho+P)=0$ and the equation of state $w_{i}(z)=P_{i} / \rho_{i}$, where the index $i$ represents the fluid species sourcing the Universe. It is then convenient to define the density parameters $\Omega_{i} \equiv \rho_{i} / \rho_{c}$, normalized to the critical density $\rho_{c} \equiv 3 H_{0}^{2} /(8 \pi G)$.

According to the observed densities, the content of the Universe can be assumed as made of dust matter and dark energy, while we can neglect the contribution of radiation at late times. Consistently with most recent cosmological observations (Aghanim et al. 2018), we also assume that the universe has vanishing curvature $(k=0)$ also if, recently, there are claims that it could be $k \neq 0$ (Di Valentino, Melchiorri \& Silk 2020) but this result has to be definitely proved. The standard strategy adopted in cosmography is to expand the scale factor in Taylor series around the present time, namely the age of the Universe $\left(t_{0}\right)$, to study the accelerated phase of the cosmic expansion ${ }^{3}$ :

$a^{(3)}=\left[1+H_{0}\left(t-t_{0}\right)-\frac{1}{2} q_{0} H_{0}^{2}\left(t-t_{0}\right)^{2}+\frac{1}{3 !} j_{0} H_{0}^{3}\left(t-t_{0}\right)^{3}\right]$

From Eq. (3), which represents the third-order expansion of the scale factor, one can define the cosmographic series, i.e. the series of coefficients in terms of derivatives of the scale factor. These terms are defined from symmetry principles (Weinberg 1972), without invoking specific solutions to Eqs. (2):

$H(t) \equiv \frac{1}{a} \frac{d a}{d t}, \quad q(t) \equiv-\frac{1}{a H^{2}} \frac{d^{2} a}{d t^{2}}, \quad j(t) \equiv \frac{1}{a H^{3}} \frac{d^{3} a}{d t^{3}}$,

3 The cosmographic technique allows to study the evolution of the Universe in a model-independent way by means of kinematic variables (Visser 2015). In other words, cosmography does not require the assumption of any specific cosmological model to investigate the behaviour of dark energy. In this picture, the equation of state of the cosmic fluid is not postulated a priori, and the only assumption concerns the validity of the cosmological principle.
These coefficients are known as Hubble, deceleration and jerk parameters. In particular, from the sign of $q$, one can infer whether the universe is decelerating, i.e. $q_{0}>0$, or accelerating, i.e. $q<0$; a positive sign of $j$ indicates a transition time between the two phases. More recently, the cosmographic series has been extended in order to compare cosmological models that degenerate with respect to $q_{0}$ and $j_{0}$. In other words, one has the need to compute more than three cosmographic coefficients to show possible deviations from the standard cosmological model. So, extending Eq. (3) up to the fifth order, we have

$a^{(5)}=a^{(3)}+\left[\frac{1}{4 !} s_{0} H_{0}^{4}\left(t-t_{0}\right)^{4}+\frac{1}{5 !} l_{0} H_{0}^{5}\left(t-t_{0}\right)^{5}\right]$,

where we have introduced

$s(t) \equiv \frac{1}{a H^{4}} \frac{d^{4} a}{d t^{4}}, \quad l(t) \equiv \frac{1}{a H^{5}} \frac{d^{5} a}{d t^{5}}$,

colled the snap and lerk parameters, respectively. The possibility to discriminate between a given model and the standard cosmological one passes through taking into account at least $a^{(4)}$ according to the present state of observations.

\subsection{The standard approach to cosmography}

With the above considerations, one can use the definition of the redshift in terms of the scale factor, $z=a^{-1}-1$, to relate the Hubble parameter to the luminosity distance $d_{L}(z)$ as

$H(z)=\left[\frac{d}{d z}\left(\frac{d_{L}(z)}{1+z}\right)\right]^{-1}$,

to obtain

$\begin{aligned} d_{L}(z) & =\frac{z}{H_{0}} \sum_{n=0}^{N} \frac{\alpha_{n}}{n !} z^{n}, \\ H(z) & =\sum_{m=0}^{M} \frac{H^{(m)}}{m !} z^{m},\end{aligned}$

or, explicitly, at the $5^{\text {th }}$ order of expansion in $d_{L}(z)$, one obtains

$d_{L}^{(5)}=\frac{z}{H_{0}}\left(\alpha_{0}+\alpha_{1} z+\alpha_{2} \frac{z^{2}}{2}+\alpha_{3} \frac{z^{3}}{6}+\alpha_{4} \frac{z^{4}}{24}\right)$,

or, at the $4^{\text {th }}$ order of expansion in $H(z)$, we have ${ }^{4}$

$\mathcal{E}^{(4)} \simeq 1+H_{1} z+H_{2} \frac{z^{2}}{2}+H_{3} \frac{z^{3}}{6}+H_{4} \frac{z^{4}}{24}$.

Therefore, by means of the definitions (4)-(6), one can write the coefficients $\alpha_{i}, H_{i}$ in terms of the cosmographic parameters (see Section 5 for details) and compare the above formulae directly with observations. The difference in the expansion order between $d_{L}$ and $H$ is due to the definition of $d_{L}$ which gives rise to some additional complexity with respect to fitting directly through $H$. For these reasons, a given order of $d_{L}(z)$ provides much more statistical discrepancies with respect to $H(z)$ of the corresponding order (Luongo 2013, 2011; Aviles et al. 2013).

${ }^{4}$ We consider $\mathcal{E} \equiv H / H_{0}$. 
This fact implies errors which are produced by the truncations and so they propagate as $\sim \mathcal{O}(6)$ and $\sim \mathcal{O}(5)$ for $d_{L}$ and $H$ respectively. In fact, systematics induced by truncating the series at a certain order may influence the cosmographic analysis, biasing the corresponding numerical results. If, from one hand, the convergence decreases when additional higher-order terms are introduced, on the other hand, the accuracy of the analysis may be compromised by considering only lower orders. A possible solution to this issue is to analyze series at different orders and thus constrain different sets of parameters, considering some sort of hierarchy among series. In the following, we will consider this approach and we will constrain different orders to check the statistical and numerical differences of the produced outcomes.

Let us conclude this section by discussing the role of spatial curvature in the cosmographic context. Degeneracy among coefficients and spatial curvature is due to the fact that all the cosmographic parameters are related to the Hubble rate. In particular, the contribution of the Hubble constant in the luminosity distance can be factorized into $d_{L}=d_{H} \times \widetilde{d}_{L}\left(z ; q_{0}, j_{0}, \ldots\right)$, where $d_{H} \equiv H_{0}^{-1}$. Then, $d_{H}$ becomes an additional constant as one fits with respect to supernovae, requiring that a single data set is unable to break the degeneracy among coefficients. The same happens when one considers spatial curvature which degenerates with $j$ and further orders, and so one cannot bound them only without fixing spatial curvature. To heal this problem, in this work, we assume $k=0$, in agreement with the CMB observations which confine its value to a very small interval around zero (Aghanim et al. 2018). The aim is to compute single coefficients instead of combinations between cosmographic series and $k$. However, although this scheme is well consolidated and works fairly, we still do not have hints on how to perform a direct high-redshift cosmography. The former is intimately related to the problem of convergence, consisting on the issue that affects the cosmographic series when data beyond the limit $z<1$ are considered. To better realize this, it is easy to notice that the Taylor series has a limited convergence radius. In the next section, we face the convergence problem and we discuss how to overcome it by extending the Taylor series up to high-redshift domains using strategies over the expansions themselves.

\section{TOWARDS A SOLUTION OF THE COSMOGRAPHIC ISSUES}

According to what discussed above, cosmographic Taylor series are expected to suffer from divergence problems as cosmic data exceed the limit $z<1$. In other words, truncating series that are obtained by expansions around $z=0$ causes difficulties to analyze data at $z \geqslant 1$. This fact leads to systematic error propagations and limits the numerical results which are affected by enhanced bad convergence. The convergence issue is the main limitation of cosmography, and so it becomes of fundamental importance overcoming it by building up some more reliable cosmographic treatment. To do so, one can employ rational polynomials or new parametrizations of the redshift variable ${ }^{5}$, with the final aim of extending the convergence radii of standard Taylor expansions. Both rational approximations and parametrizations of $z$ are artificial and need specific techniques to be taken into account.

We now show that, albeit widely used to overcome the convergence problem, auxiliary variables are much less predictive than particular classes of rational approximations. In particular, we confront both the approaches showing that auxiliary variables are not adequate to deal with cosmological data at high redshifts, although they have been introduced with this purpose.

\subsection{First solution: rational polynomials}

Making cosmographic expansions stable at high redshifts can be possible as one assumes $(n, m)$ rational approximations of a given cosmological observable. Indeed, approximations are built from the ratio between a $n$-th and a $m$-th order polynomials, leading to a overall order, $n+m$, which univocally defines the set of parameters entering cosmological fits. The construction of rational approximations is jeopardized by the issue of degeneracy among the cosmographic coefficients. To picture this fact, let us take as an example $d_{L}^{(n=2)}(m=1)$. In terms of Taylor expansions, it leads to a thirdorder approximation defined by three cosmographic parameters. However, there exists a degeneration between the orders, as also $d_{L}^{(n=1)}(m=2)$ is equivalent to its Taylor analogue, albeit $d_{L}^{(m=2)}\left(\begin{array}{l}(n=1) \\ (m)\end{array} d_{L}^{(n=1)}\right)$ are different between them.

This fact becomes crucial when high redshift data are involved. In fact, for small redshifts, rational expressions are essentially indistinguishable to the Taylor one, but, as highredshift surveys are considered, the situation dramatically changes. Here we propose a first criterion that one should consider to choose the correct rational approximation. We need the convergence radius of rational polynomials to be equivalent or higher than the corresponding Taylor series, determining the following practical rule:

- The most suitable rational approximation corresponds to the function that maximizes the convergence radius, once the orders $(n, m)$ are chosen.

The advantage is easy to understand: the stability of the approximation is intimately related to its convergence radius, and thus stable rational approximations would give stable fitting procedures. In principle, however, this is valid for an infinite number of parameters only, namely as the original function is fully-recovered. As a consequence, this issue provides a second practical rule:

- The most suitable rational approximation is the one that minimizes the number of parameters of $m$, taking into account the first condition mentioned above.

In fact, the more parameters are in the denominator, the larger is the freedom (degeneration) in the choice of intervals that can nullify the denominator, creating poles. When the number of parameters is small, one can better handle this situation. In other words, the denominator, i.e. the only part

5 This is possible by means of auxiliary variables, which maps the domain $z \in(0, \infty)$ into a sphere of convergence radius $R_{\rho} \leqslant 1$. 
of rational approximations which can create poles, might be stable. Thus, a small number of free parameters is essential to enable the rational approximation to be convergent. The two aforementioned conditions translate in finding the best compromise between arbitrary-order expansions and minimal number of free parameters in the denominator.

Likely the simplest choice is then to consider the $(2,1)$ rational polynomial characterized by only one parameter in the denominator, i.e. minimizing $m$ as the overall order is fixed. However, this provides additional issues in the choice of the best polynomials. In fact, other choices, e.g. $(3,1)$ and $(4,1)$ polynomials, are equally valid, in principle, and in the following we ought to explain why the $(2,1)$ polynomials should be preferred over the $(3,2)$ or the $(1,1)$ and $(2,2)$ and so forth from a statistical point of view.

\subsubsection{The Padé approximation}

As a relevant example, we present the method of the Padé approximations (Krantz \& Parks 1992). The Padé technique is built up from the standard Taylor definition and is used to lower divergences at $z \geqslant 1$. Thus, a given function $f(z)=$ $\sum_{i=0}^{\infty} c_{i} z^{i}$, expanded with a given set of coefficients, namely $c_{i}$, is approximated by means of a $(n, m)$ Padé approximant by the ratio

$$
P_{n, m}(z)=\frac{\sum_{i=0}^{n} a_{i} z^{i}}{1+\sum_{j=1}^{m} b_{j} z^{j}},
$$

where the Taylor expansion matches the coefficients of the above expansion up to the highest possible order:

$$
\begin{aligned}
& P_{n, m}(0)=f(0), \\
& P_{n, m}^{\prime}(0)=f^{\prime}(0), \\
& \vdots \\
& P_{n, m}^{(n+m)}(0)=f^{(n+m)}(0) .
\end{aligned}
$$

By construction, in the numerator we have $n+1$ independent coefficients, whereas in the denominator $m$, for a total of $n+m+1$ unknown terms. From

$$
\sum_{i=0}^{\infty} c_{i} z^{i}=\frac{\sum_{i=0}^{n} a_{i} z^{i}}{1+\sum_{j=1}^{m} b_{j} z^{j}}+\mathcal{O}\left(z^{n+m+1}\right),
$$

and, then,

$\left(1+b_{1} z+\ldots+b_{m} z^{m}\right)\left(c_{0}+c_{1} z+\ldots\right)=a_{0}+a_{1} z+\ldots+a_{n} z^{n}+\mathcal{O}\left(z^{n}\right.$

equating the same power coefficients, one gets $n+m+1$ equations with $n+m+1$ free variables. The advantages of Padé rational approximations are thus:

- the polynomials approximate flexes and singular points in a better way than Taylor;

- the polynomials reduce error bias propagating as data are outside the limit $z<1$;
- the polynomials, independently from choosing appropriate orders, hold for all terms to fit in the numerator.

Hence, Padé polynomials are expected to extend the convergence radius of the Taylor series. Another relevant fact is that Padé series, among all rational approximations, address in a simple way how to choose the orders $(n, m)$. In fact, we note that in cosmography we approximate a generic function $f$ as $f \sim f_{0}+f_{1}+f_{2}+f_{3}+\ldots$, where $\left|f_{3}=f_{3}\left(q_{0}, j_{0}\right)\right|<\left|f_{2}\left(q_{0}\right)\right|$, due to the larger weight of the lowest orders with respect to the higher orders reflecting the lower stability of the less numerous high-redshift data. This implies that a rational function of the type $x /\left(1+x+x^{2}\right)$ approximatively behaves as $x /(1+x)$, or $\left(x+x^{2}\right) /(1+x) \sim$ $x+x^{2}$ and so on, with $x$ an arbitrary analytical variable. Numerator and denominator of the same order cause induced errors in the analysis as, for $x \rightarrow \infty, N / D \rightarrow$ const, where $N=N(x)$ and $M=M(x)$ are the polynomials in the numerator and denominator, respectively. This is the reason why rational approximations with the same order in the numerator and denominator do not provide accurate cosmographic results.

Understanding why the $(2,1)$ polynomial is preferred over the $(3,2)$ and $(3,1)$ orders is related to the degeneracy among coefficients. By construction, in the Padé series, all the free parameters are present already in the numerator. Padé series are of lower order than the corresponding Taylor polynomials. As an example, we mention that for $(3,1)$ and $(2,1)$ polynomials, one has respectively

$P_{3,1}(z)=\frac{a_{0}+a_{1} z+a_{2} z^{2}+a_{3} z^{3}}{1+b_{1} z}$,

and

$P_{2,1}(z)=\frac{a_{0}+a_{1} z+a_{2} z^{2}}{1+b_{1} z}$.

In the $(3,1)$ case, we have $\left|b_{1}\right| \ll\left|a_{1}\right|$ and $\left|b_{1}\right| \ll\left|a_{2}\right|$, while in the $(2,1)$ case, we have $\left|a_{1}\right| \sim\left|a_{2}\right| \cdot\left|b_{1}\right|$. As a result, in the $(3,1)$ case, the denominator does not enter the problem, whereas it does play a role in the $(2,1)$ case. Therefore, at low redshifts, the numerical fits are influenced by all the parameters in the $(2,1)$ case, contrary to the $(3,1)$ case. This provides us with the following rule:

- The rational approximation is, for an arbitrary choice of $z$ (let us say $z=1$ for simplicity), a Padé series in which the ratio maximizes the condition $\left.\frac{N}{D}\right|_{z=1} \approx 1$, and is not $\gg 1$.

The above condition is clearly verified for any order, also for the $(2,1)$ case. This implies that

- error bars increase as low-redshift data only are involved;

- error bars decrease as high-redshift data are included.

In fact, adding high-redshift data makes the denominator count more than the numerator, thus minimizing the errors with respect to the order. We can then summarize our findings as follows:

- The Padé series are suitable tools to address highredshift cosmography, since they involve rational polynomials which account for the aforementioned conditions. 
- The most stable order of the Padé series is (2,1); we can demonstrate this statement based on the constructions of Padé series and on mathematical rules derived from the degeneracy among coefficients.

- The Padé series are characterized by larger uncertainties compared to the corresponding Taylor series. This is evident as much as small data are involved into the problem, while becomes non-relevant as high-redshift data are involved. Unfortunately sets of cosmic data surveys with $z>1$ are typically poorly constrained, leaving cosmography non-predictive if only high-redshift data are included into experimental analyses.

- The errors associated to the Padé series reduce as soon as high-redshift data are included into the analysis. The explanation derives from the previous point: in the case of high-redshift data only, if the data set is large, Padé series become stable and permit a high-redshift cosmography at arbitrary redshift.

To summarize, the problem of constructing a modelindependent cosmography consists in adopting a rational approximant built up in terms of Padé polynomial, which fulfills all the aforementioned requirements. In particular, the Padé approximation that guarantees the best outcomes in the fitting procedures is given by the $(2,1)$ polynomial. This leads to the problem that no more than $s_{0}$ can be accurately fitted even with higher redshift data, in agreement with previous works (Cattoen \& Visser 2008). The question left open is therefore: does it exist an alternative strategy toward the determination of better convergent cosmographic series? Let us discuss this point by using auxiliary variables in the next section.

\subsection{Second solution: auxiliary variables}

Another approach to heal the convergence problem is represented by the use of auxiliary $y$-variables. To build up a generic parametrization of $y$, we can write

$y=\mathcal{F}(z)$,

satisfying the conditions

$$
\begin{aligned}
\mathcal{F}(\infty) & <\infty, \\
\mathcal{F}(0) & =\mathcal{C}<\infty .
\end{aligned}
$$

The properties (22)-(23) are fundamental to construct a $y$-variable that reduces the convergence radius when $z \rightarrow$ $\infty$. The procedure to reformulate the luminosity distance, $d_{L}(y)$, in terms of Eq. (21) is possible in two ways. The first, after Taylor-expanding around $z=0$ the function $d_{L}(z)$, consists in substituting $z$ in terms of $y$ to get $\widetilde{d}_{L}\left(\mathcal{F}^{-1}(y)\right)$. Then, expanding in series around $y(0)$ permits to get $d_{L}(y)$. This implies that the most suitable choice of $\mathcal{C}$ is $\mathcal{C}=0$, fulfilling the fact that our cosmic era is characterized by $z=\mathcal{F}=0$. The second procedure takes into account an exact version of $d_{L}(y)$ without expanding it, as before, around $z=0$. Only after this assumption, one can expand the exact luminosity distance, obtaining the same result of the first method. The equivalence of the two approaches is due to the analyticity of $d_{L}(z)$ and justifies why, at $z=0$, we should have $y=0$. From a theoretical point of view, the auxiliary variables are unable to reproduce the $\Lambda$ CDM predictions, being unable to be suitable alternatives to Taylor expansions in general. To see this fact, it is enough to notice that when $z \rightarrow \infty$, we have $\mathcal{F}<\infty$. So, the luminosity distance converges to a plateau value which departs of several orders of magnitudes, depending on the choice of $y$, from observations. To overcome this fact, an immediate example is offered by powers of $y$, say $y^{n}$ with $0<n<1$, that clearly converge less rapidly than $y$, but leave the caveat toward the understanding of the most suitable order $n$, introducing a new problem: inferring the best $n$ from observations.

So that, in principle, one can imagine to re-parametrize the redshift with a function that abolishes the second condition displayed in Eq. (23). An example has been considered in Risaliti \& Lusso (2015, 2019), where the authors introduced a $\log$-polynomial $y$-variable. In such a way, they expanded the series around $\log _{10}(1+z)$, instead of $z$. Although intriguing, this only leads to re-parametrizing $z$, instead of a robust and well-motivated approach against the convergence problem, leaving a cautionary tale for cosmographic applications at $z \rightarrow \infty$. In other words, if one does not take into account the second condition, Eq. (23), it is not possible to alleviate in any cases the convergence issue. At small and intermediate redshifts, the use of auxiliary variables is impracticable since errors increase dramatically, with no substantial improvements on the cosmographic coefficients that are still far from being predictive in disentangling dark energy from a pure cosmological constant.

Considering the standard approach of auxiliary variables, the basic assumptions to construct any pictures of $y$-cosmography are: the auxiliary variable must be one-toone invertible when passing from the redshift to it; it should not exhibit any divergence feature for any value of $z$; any new parametrization should be smooth along the Universe evolution and no critical points have to appear as the Universe expands. Relevant examples of this approach are given by Cattoen \& Visser (2007); Aviles et al. (2012). They are

$y_{1}=1-a$

$y_{2}=\arctan \left(1-a^{-1}\right)$.

When these choices are plugged into $d_{L}$, without a series expansion, one typically gets sources of errors. For example, avoiding the series expansion after considering the change $z=\mathcal{F}^{-1}(y)$ in $d_{L}$ produces a rational function that cannot be better than parametrizing the redshift by means of Padé approximations. This is why the formers behave better, i.e. they can be interpreted as convergent classes of auxiliary variables. This argument can be used to explain why $y=$ $\arctan (z)$ represents a better parametrization than $y_{1}$ and than any powers of $y_{1}$. In fact, this function is not a rational polynomial of $z$ built up ad hoc, but a real function, as well as the log-parametrization that has the advantage to converge at very large redshifts. Even in this case the convergence creates a plateau at $z \rightarrow \infty$ and so does not explain very well the Universe expansion history at all times, but only at small and intermediate redshifts. In general, we can conclude with the following statement:

- The most suitable parametrization is the one that most closely reproduces $z$ at any intervals of the Universe evolution.

Moreover, by means of similar arguments, to contrive a viable redshift parametrization, the following conditions must be satisfied: 
(i) The luminosity distance curve should not behave too steeply in the interval $z<1$.

(ii) The luminosity distance curve should not exhibit sudden flexes.

(iii) The curve should be one-to-one invertible.

The last three requirements are fulfilled for both $y_{1}$ and $y_{2}$. The second auxiliary variable, $y_{2}$, works better than the previous one. We are now ready to compare $y$-cosmography with Padé approximations directly with cosmic data.

\section{OBSERVATIONAL CONSTRAINTS AND THE BAYESIAN INFERENCE}

To analyze the stability and convergence of the different cosmographic techniques, let us consider the approximations of the luminosity distance based on $^{6}$ :

- Taylor expansion of the third order $\left(T_{3}\right)$, fourth order $\left(T_{4}\right)$ and fifth order $\left(T_{5}\right)$;

- $y$-redshift expansions of the third order $\left(y_{i}^{(3)}\right)$, fourth order $\left(y_{i}^{(4)}\right)$ and fifth order $\left(y_{i}^{(5)}\right)$ for the variables $y_{1}$ and $y_{2}$;

- rational Padé polynomials of the third order $\left(P_{2,1}\right)$, fourth-order $\left(P_{2,2}\right)$ and fifth order $\left(P_{3,2}\right)$. These specific polynomials have been chosen according to their good behaviours over a large interval of redshift (cf. Figure 1).

As a guideline, we use the predictions of the flat $\Lambda \mathrm{CDM}$ model, characterized by the following Hubble expansion rate:

$H_{\Lambda \mathrm{CDM}}(z)=H_{0} \sqrt{\Omega_{r 0}(1+z)^{4}+\Omega_{m 0}(1+z)^{3}+1-\Omega_{r 0}-\Omega_{m 0}}$,

where $\Omega_{r 0} \approx 5 \times 10^{-5}$ is the radiation density and $\Omega_{m 0}$ is the present matter density parameter.

From the definitions (4)-(6) and Eq. (25), it is possible to relate the cosmographic parameters to the physical quantity $\Omega_{m 0}$ as

$q_{0}=-1+\frac{3}{2} \Omega_{m 0}$,

$j_{0}=1$,

$s_{0}=1-\frac{9}{2} \Omega_{m 0}$

$l_{0}=1+3 \Omega_{m 0}-\frac{27}{2} \Omega_{m 0}^{2}$.

Moreover, assuming the concordance value $\Omega_{m 0}=0.3$, one obtains the "fiducial set":

$\left(q_{0}, j_{0}, s_{0}, l_{0}\right)=(-0.55,1,-0.35,0.685)$.

We therefore split our analysis in two stages, i.e. low and high-redshift domains.

6 We refer the readers to Section 5 for the explicit expressions of the luminosity distance and the Hubble expansion rate corresponding to the different cosmographic techniques.

\subsection{Low-redshift domain}

First, we considered the low-redshift regime through the use of the Pantheon type Ia Supernovae (SNe Ia) catalogue (Scolnic et al. 2018) and the observational Hubble data (OHD) acquired by means of the differential age method (Jimenez \& Loeb 2002). We refer the readers to D'Agostino (2019) and references therein for details on both SNe Ia and OHD data sets and their respective likelihood functions. We thus performed a Monte Carlo Markov Chain (MCMC) integration on the combined SNe Ia+OHD likelihood. Table 1 summarizes the numerical results for the cosmographic parameters up to the $95 \%$ confidence level (C.L.). It is interesting to compare these values with the values derived from Eqs. (26)-(29) after fitting the $\Lambda$ CDM model to the data. In particular, for the $\mathrm{SNe} \mathrm{Ia}+\mathrm{OHD}$ analysis, we find $\Omega_{m 0}=0.295_{-0.029(0.051)}^{+0.02(0.058)}$, leading to the derived values reported in Table 1 for the $\Lambda$ CDM model. Moreover, in Figure 2 , we show the $1 \sigma$ and $2 \sigma$ contours in the 2 -D parameter space divided by orders of expansion.

\subsection{High-redshift domain}

To check also the high-redshift behaviour of our approximations on $d_{L}(z)$, we then take into account the CMB measurements by means of the shift parameter (Efstathiou \& Bond 2005):

$\mathcal{R} \equiv H_{0} \sqrt{\Omega_{m 0}} \frac{d_{L}\left(z_{r e c}\right)}{\left(1+z_{r e c}\right)}$,

which involves the luminosity distance at the epoch of recombination $\left(z_{\text {rec }}\right)$. The parameter $\mathcal{R}$ is effectively model-independent and insensitive to perturbations (Maartens \& Majerotto 2006), and in our analysis we used the estimates of the Planck collaboration (Ade et al. 2016), namely $\mathcal{R}=1.7488 \pm 0.0074$, together with $z_{\text {rec }}=1090.09$ and $\Omega_{m 0}=0.315$. The results emerging from the combination of low and high-redshift data $(\mathrm{SNe} \mathrm{Ia}+\mathrm{OHD}+\mathcal{R})$ are summarized in Table 2 up to the $95 \%$ C.L., while Figure 3 shows the $1 \sigma-2 \sigma$ contours in the $2-\mathrm{D}$ parameter space. In this case, the reference $\Lambda \mathrm{CDM}$ values of the cosmographic parameters are derived from the measured value $\Omega_{m 0}=0.317_{-0.003(0.007)}^{+0.003(0.007)}$.

\subsection{Statistical analysis and selection criteria}

Afterwards, we analyze the statistical performances of the various cosmographic approaches through the use of Bayesian selection criteria to measure the evidence of a given model against a reference scenario (Kunz, Trotta \& Parkinson 2006), which we chose to be the standard $\Lambda$ CDM model. Specifically, we considered the Akaike information criterion (AIC) (Akaike 1974) and the Bayesian information criterion (BIC) (Schwarz 1978), defined as, respectively,

$\mathrm{AIC} \equiv-2 \ln \mathcal{L}_{\max }+2 p$,

$\mathrm{BIC} \equiv-2 \ln \mathcal{L}_{\max }+p \ln N$.

Here, $\mathcal{L}_{\max }$ is the maximum likelihood estimate, $p$ is the number of free parameters of the model and $N$ the total number of data points. We note that, for high $N$, the BIC 


\begin{tabular}{|c|c|c|c|c|c|c|c|}
\hline Model & $H_{0}$ & $q_{0}$ & $j_{0}$ & $s_{0}$ & $l_{0}$ & $\Delta \mathrm{AIC}$ & $\Delta \mathrm{BIC}$ \\
\hline$\Lambda \mathrm{CDM}$ & $69.2_{-1.9(3.8)}^{+1.9(3.8)}$ & $-0.56_{-0.04(0.08)}^{+0.04(0.09)}$ & 1 & $-0.33_{-0.12(0.23)}^{+0.13(0.26)}$ & $0.71_{-0.14(0.25)}^{+0.13(0.29)}$ & 0 & 0 \\
\hline$T_{3}$ & $69.2_{-2.0(3.8)}^{+2.0(3.9)}$ & $-0.58_{-0.08(0.15)}^{+0.08(0.16)}$ & $1.02_{-0.24(0.46)}^{+0.24(0.49)}$ & - & - & 1.28 & 2.89 \\
\hline$T_{4}$ & $69.3_{-2.0(3.9)}^{+2.0(3.9)}$ & $-0.66_{-0.13(0.25)}^{+0.13(0.26)}$ & $1.58_{-0.83(1.58)}^{+0.83(1.63)}$ & $0.97_{-1.88(2.76)}^{+1.15(3.54)}$ & - & 2.47 & 5.69 \\
\hline$T_{5}$ & $\begin{array}{r}69.3_{-2.0(3.8)}^{+2.0(3.9)} \\
\end{array}$ & $-0.58_{-0.10(0.20)}^{+0.10(0.21)}$ & $0.98_{-0.48(0.99)}^{+0.54(0.88)}$ & $-0.63_{-1.57(2.64)}^{+1.49(2.67)}$ & $4.94_{-2.12(4.75)}^{+2.53(4.27)}$ & 2.98 & 7.81 \\
\hline$y_{1}^{(3)}$ & $69.8_{-2.0(3.9)}^{+2.0(4.0)}$ & $-1.10_{-0.16(0.30)}^{+0.16(0.30)}$ & $8.08_{-1.50(2.76)}^{+1.49(2.92)}$ & - & - & 16.5 & 18.1 \\
\hline$y_{1}^{(4)}$ & $69.6_{-2.1(4.1)}^{+2.1(4.2)}$ & $-0.30_{-0.11(0.21)}^{+0.11(0.21)}$ & $0.26_{-1.17(2.58)}^{+1.39(2.39)}$ & $7.49_{-4.83(8.17)}^{+4.63(8.33)}$ & - & 19.4 & 22.6 \\
\hline$y_{1}^{(5)}$ & $69.5_{-2.0(3.8)}^{+2.0(3.9)}$ & $-0.75_{-0.11(0.21)}^{+0.11(0.23)}$ & $2.30_{-0.87(1.82)}^{+0.90(1.68)}$ & $0.21_{-4.07(7.72)}^{+4.47(7.80)}$ & $0.64_{-2.32(5.27)}^{+2.65(4.86)}$ & 4.59 & 9.42 \\
\hline$y_{2}^{(3)}$ & $69.7_{-2.0(3.7)}^{+2.0(3.9)}$ & $-0.81_{-0.10(0.20)}^{+0.10(0.20)}$ & $2.82_{-0.49(0.89)}^{+0.49(0.99)}$ & - & - & 7.71 & 9.32 \\
\hline$y_{2}^{(4)}$ & $69.4_{-1.9(3.8)}^{+1.9(3.8)}$ & $-0.59_{-0.17(0.32)}^{+0.17(0.33)}$ & $0.56_{-1.59(2.69)}^{+1.59(2.72)}$ & $-3.58_{-3.13(4.80)}^{+2.11(5.83)}$ & - & 1.44 & 4.66 \\
\hline$y_{2}^{(5)}$ & $69.2_{-2.0(3.9)}^{+2.0(4.0)}$ & $-0.55_{-0.16(0.29)}^{+0.14(0.31)}$ & $0.54_{-0.78(1.91)}^{+0.96(1.72)}$ & $3.46_{-1.25(2.19)}^{+0.83(2.69)}$ & $\begin{array}{r}4.64_{-2.47(4.55)}^{+2.29(4.62)} \\
\end{array}$ & 3.44 & 8.27 \\
\hline$P_{2,1}$ & $69.3_{-2.0(3.8)}^{+2.0(3.9)}$ & $-0.73_{-0.13(0.26)}^{+0.13(0.26)}$ & $2.84_{-1.23(2.09)}^{+1.00(2.28)}$ & - & - & 1.20 & 2.81 \\
\hline$P_{2,2}$ & $69.1_{-2.0(3.8)}^{+2.0(3.9)}$ & $-0.60_{-0.10(0.19)}^{+0.10(0.21)}$ & $1.53_{-0.81(1.57)}^{+0.81(1.59)}$ & $4.15_{-5.29(7.52)}^{+3.36(9.26)}$ & - & 3.17 & 6.39 \\
\hline$P_{3,2}$ & $69.1_{-1.9(3.8)}^{+1.9(3.8)}$ & $-0.60_{-0.10(0.19)}^{+0.10(0.19)}$ & $1.32_{-0.63(1.21)}^{+0.57(1.27)}$ & $8.47_{-2.28(3.80)}^{+1.52(4.56)}$ & $-2.1_{-4.3(16.4)}^{+7.7(11.8)}$ & 2.59 & 7.42 \\
\hline
\end{tabular}

Table 1. MCMC results at the $68 \%$ (95\%) C.L. for different cosmographic techniques from the low-redshift (SNe Ia+OHD) probes, compared to the derived predictions of the flat $\Lambda$ CDM model. $H_{0}$ values are expressed in $\mathrm{km} / \mathrm{s} / \mathrm{Mpc}$. The AIC and BIC values are computed with respect to the reference $\Lambda \mathrm{CDM}$ model.

\begin{tabular}{|c|c|c|c|c|c|c|c|}
\hline Model & $H_{0}$ & $q_{0}$ & $j_{0}$ & $s_{0}$ & $l_{0}$ & $\Delta \mathrm{AIC}$ & $\Delta \mathrm{BIC}$ \\
\hline$\Lambda \mathrm{CDM}$ & $68.2_{-1.5(2.9)}^{+1.5(2.9)}$ & $-0.525_{-0.005(0.010)}^{+0.005(0.010)}$ & 1 & $-0.425_{-0.015(0.030)}^{+0.015(0.030)}$ & $0.596_{-0.018(0.036)}^{+0.018(0.037)}$ & 0 & 0 \\
\hline$y_{1}^{(3)}$ & $74.8_{-2.2(4.4)}^{+2.2(4.2)}$ & $-1.92_{-0.07(0.13)}^{+0.07(0.13)}$ & $15.1_{-1.3(2.4)}^{+1.3(2.4)}$ & - & - & 94.9 & 96.5 \\
\hline$y_{1}^{(4)}$ & $68.3_{-1.8(3.5)}^{+1.8(3.6)}$ & $-0.27_{-0.10(0.20)}^{+0.10(0.19)}$ & $1.92_{-0.64(1.18)}^{+0.64(1.28)}$ & $4.01_{-3.76(6.06)}^{+2.81(6.88)}$ & - & 58.2 & 61.5 \\
\hline$y_{1}^{(5)}$ & N.D. & N.D. & N.D. & N.D. & N.D. & N.D. & N.D. \\
\hline$y_{2}^{(3)}$ & $80.3_{-2.4(4.6)}^{+2.4(4.6)}$ & $-2.31_{-0.03(0.05)}^{+0.03(0.05)}$ & $11.23_{-0.37(0.71)}^{+0.37(0.73)}$ & - & - & 695 & 697 \\
\hline$y_{2}^{(4)}$ & $77.6_{-1.9(3.6)}^{+1.9(3.6)}$ & $-0.80_{-0.05(0.10)}^{+0.05(0.10)}$ & $-0.63_{-0.37(0.82)}^{+0.43(0.75)}$ & $-8.28_{-1.43(3.28)}^{+1.75(2.99)}$ & - & 100 & 103 \\
\hline$y_{2}^{(5)}$ & $69.9_{-2.2(4.0)}^{+2.2(4.0)}$ & $-0.54_{-0.18(0.26)}^{+0.13(0.31)}$ & $0.12_{-0.83(1.56)}^{+0.83(1.59)}$ & $4.30_{-0.61(1.17)}^{+0.54(1.35)}$ & $-0.01_{-1.8(3.5)}^{+1.8(3.5)}$ & 2.95 & 7.86 \\
\hline$P_{2,1}$ & $70.6_{-1.9(3.7)}^{+1.9(3.8)}$ & $-0.56_{-0.09(0.21)}^{+0.11(0.18)}$ & $1.15_{-0.53(0.87)}^{+0.38(1.00)}$ & - & - & 9.67 & 11.3 \\
\hline$P_{2,2}$ & N.C. & N.C. & N.C. & N.C. & - & N.C. & N.C. \\
\hline$P_{3,2}$ & $70.9_{-2.0(3.8)}^{+2.0(3.9)}$ & $-0.57_{-0.10(0.21)}^{+0.10(0.21)}$ & $1.11_{-0.55(0.96)}^{+0.45(1.10)}$ & $2.12_{-2.62(4.12)}^{+1.51(5.68)}$ & $-0.14_{-1.03(2.09)}^{+1.10(1.83)}$ & 9.39 & 14.3 \\
\hline
\end{tabular}

Table 2. MCMC results at the $68 \%$ (95\%) C.L. for different cosmographic techniques from the combination of low and high-redshift $(\mathrm{SNe} \mathrm{Ia}+\mathrm{OHD}+\mathcal{R})$ data, compared to the derived predictions of the flat $\Lambda \mathrm{CDM}$ model. N.D. (i.e. not defined) indicates that the data are unable to constrain the parameters, whereas N.C. means no convergence of the numerical algorithm. $H_{0}$ values are expressed in $\mathrm{km} / \mathrm{s} / \mathrm{Mpc}$. The AIC and BIC values are computed with respect to the reference $\Lambda$ CDM model. 


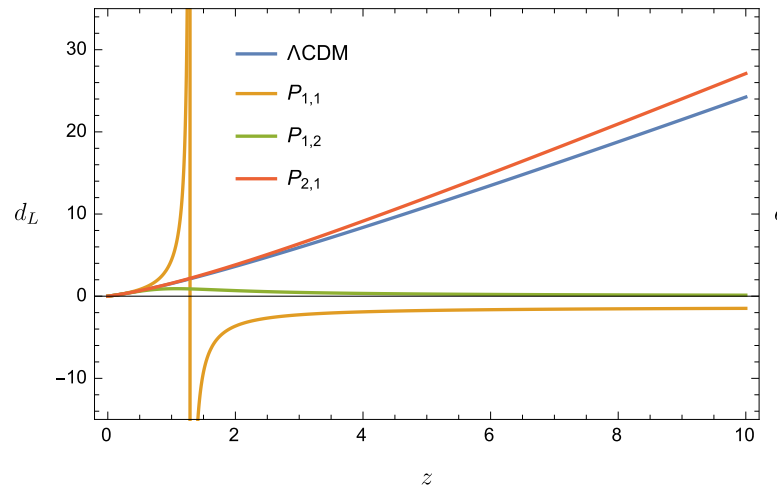

(a) Padé of orders $n+m=(2 ; 3)$

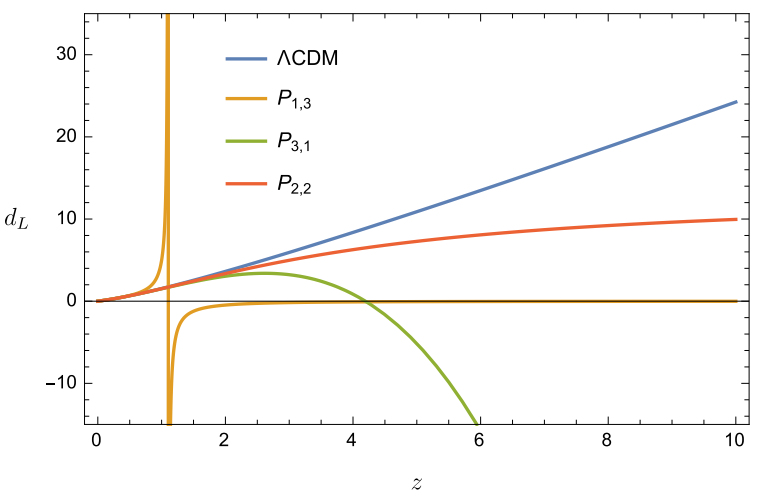

(b) Padé of orders $n+m=4$

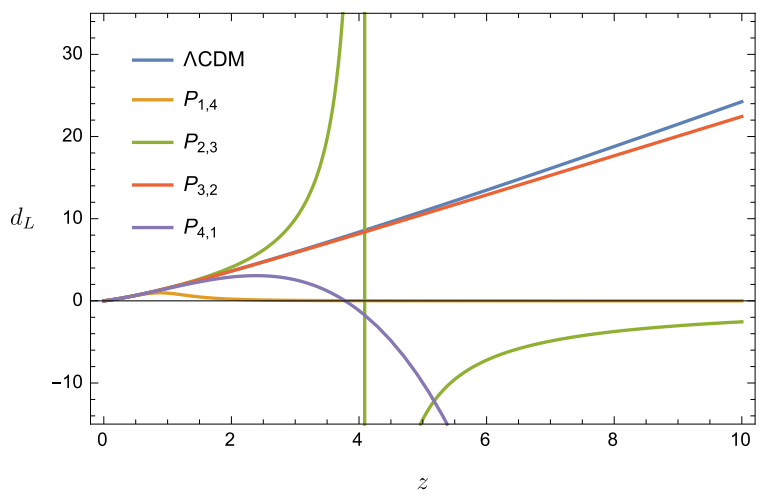

(c) Padé of orders $n+m=5$

Figure 1. Redshift evolution of the Padé approximations of the luminosity distance (in units of $H_{0}^{-1}$ ) up to the fifth order, using the fiducial set (30). The prediction of the flat $\Lambda$ CDM model is shown for comparison.

criterion penalizes more severely than AIC the model with a large number of free parameters.

Using these definitions, we calculated the differences $\triangle \mathrm{AIC}$ and $\triangle \mathrm{BIC}$ with respect to the corresponding $\Lambda \mathrm{CDM}$ values to measure the amount of information lost by adding extra parameters in the statistical fitting. Negative values of $\triangle \mathrm{AIC}$ and $\triangle \mathrm{BIC}$ indicate that the model under investigation performs better than the reference model, while for positive values, one can refer to:

- $\triangle \mathrm{AIC}(\mathrm{BIC}) \in[0,2]$ indicates a weak evidence in favour of the reference model, leaving open the question on which model is the most suitable one;

- $\triangle \mathrm{AIC}(\mathrm{BIC}) \in(2,6]$ indicates a mild evidence against the given model with respect to the reference paradigm;

- $\triangle \mathrm{AIC}(\mathrm{BIC})>6$ indicates a strong evidence against the given model, which should be rejected.

We report the $\triangle \mathrm{AIC}$ and $\Delta \mathrm{BIC}$ values for the cosmographic models in the low-redshift regime in Table 1, and for the combined low and high-redshift regimes in Table 2.

\subsection{Discussion on numerical results}

Let us now discuss the results we have obtained from the numerical analyses at low and high redshifts. It is worth noticing that the introduction of $y$-variables fails to be predictive and turns out to be a non-viable technique, excepting for a peculiar case built up in terms of $y_{2}$. In particular, in the lowredshift regime, we note that the results of the third-order $y_{1}$-variable are substantially different than $2 \sigma$ away from the corresponding Taylor constraints. The former results are the tightest and fully compatible with the $\Lambda$ CDM predictions (see top-left panel of Figure 2). However, as suggested by the BIC criterion, the $(2,1)$ Padé polynomial turns out to be the best-performing third-order approximation (cf. Table 1). At the fourth-order expansion, still the $y_{2}$-variable behaves better than the $y_{1}$-variable and provides constraints on the cosmographic parameters that are in agreement with those of the $\Lambda$ CDM model within the $68 \%$ C.L. (see top-right panel of Figure 2). The statistical evidence against the $(2,2)$ Padé polynomial with respect to $\Lambda \mathrm{CDM}$ is higher compared to the $(2,1)$ Padé polynomial (cf. Table 1). At the fifth-order expansion, we clearly note that all the techniques are characterized by large uncertainties on the parameter $l_{0}$ (see bottom panel of Figure 2). Statistically, the best model is provided by the $(3,2)$ Padé polynomial, whose AIC and BIC values are lower than the corresponding $y$-variables expansions (cf. Table 1). Hence, already in the case of small redshifts, the approximations made by means of Padé polynomials, if accurately calibrated, behave much better in matching the $\Lambda$ CDM predictions and in fixing numerical constraints over the cosmographic series. Even the error bars seem to be smaller than the ones obtained from the use of $y$-variables.

This fact is confirmed introducing high-redshift data sets. Indeed, if also the high-redshift measurements are considered, the Taylor polynomials fail to be predictive, as theoretically expected. In this case, the MCMC analysis, at the third-order expansion, indicates that the results of the 


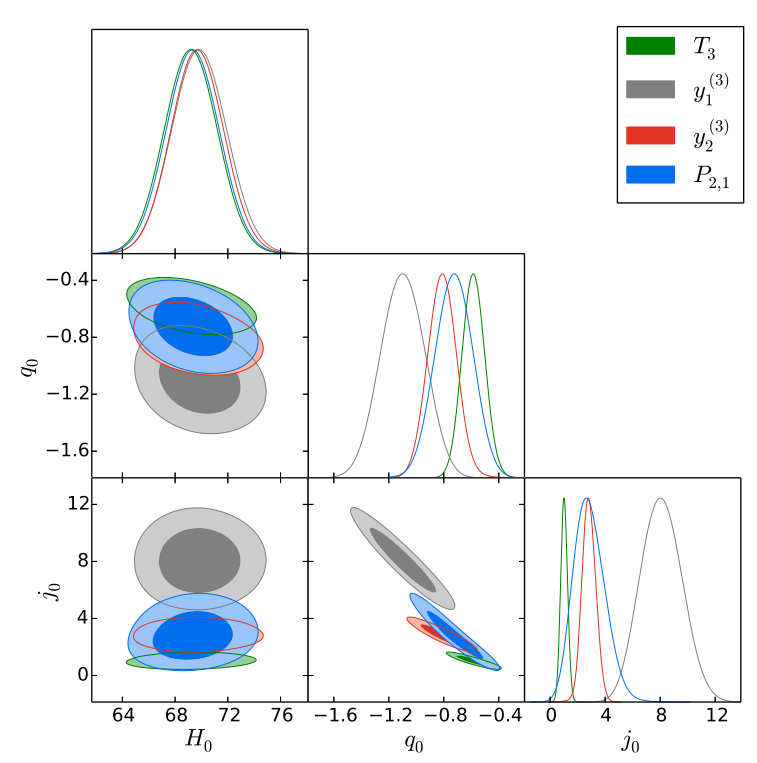

(a) Third-order analyses

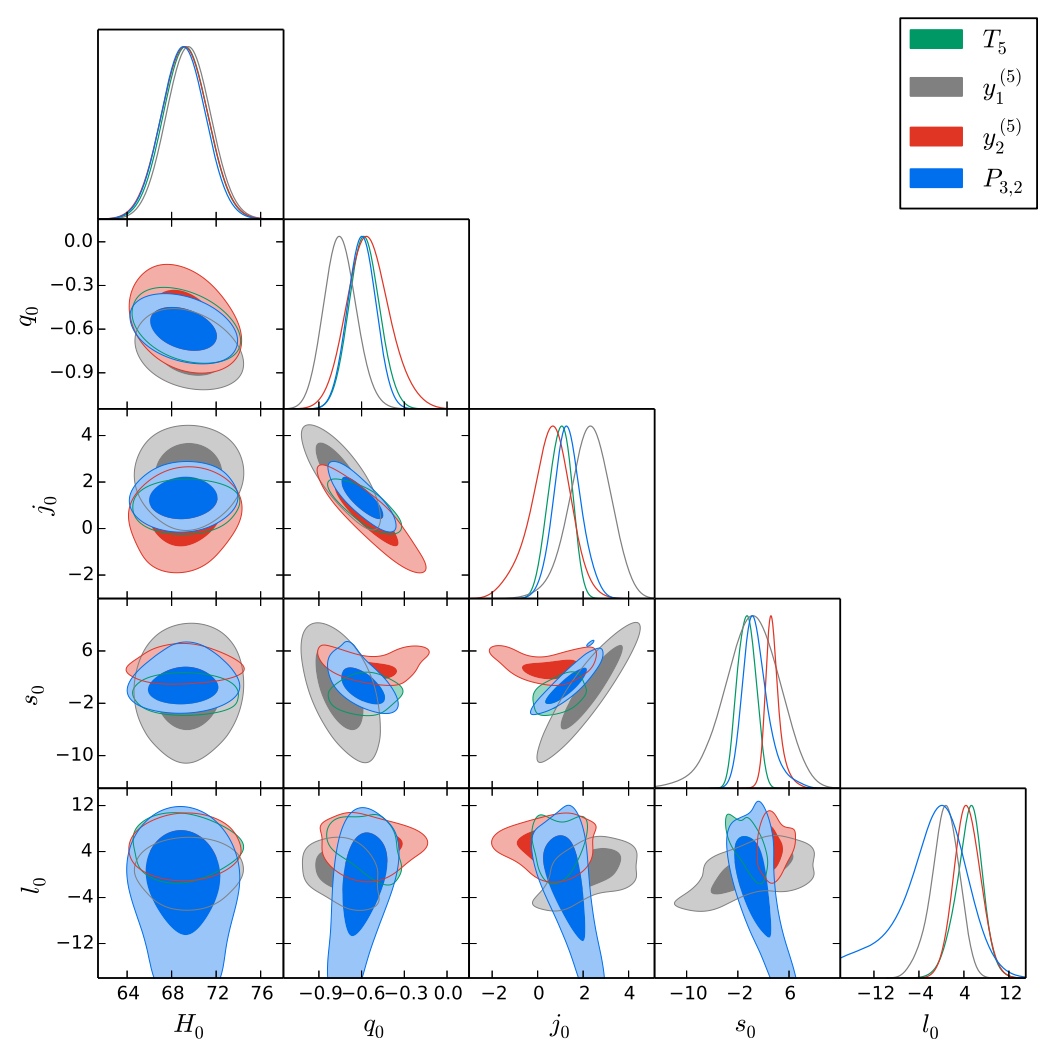

(c) Fifth-order analyses

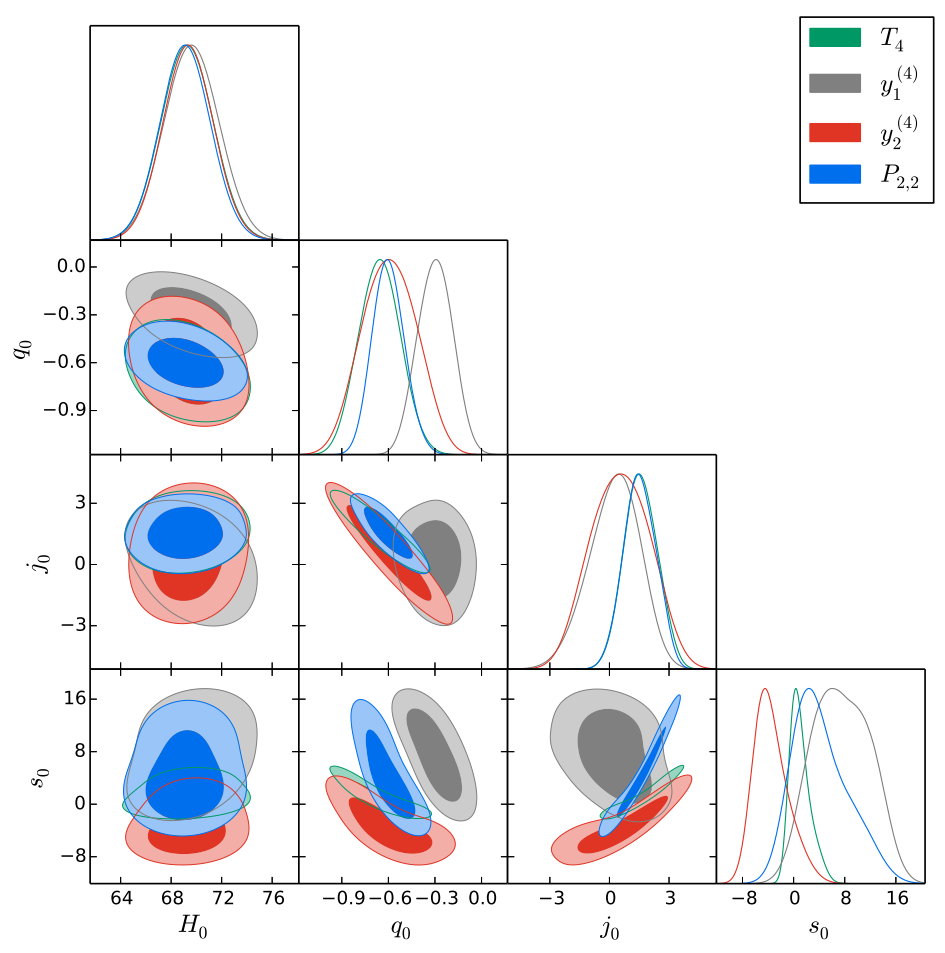

(b) Fourth-order analyses

Figure 2. Low-redshift (SNe Ia+OHD) constraints on the cosmographic parameters at the $68 \%$ and $95 \%$ C.L. for different techniques. 


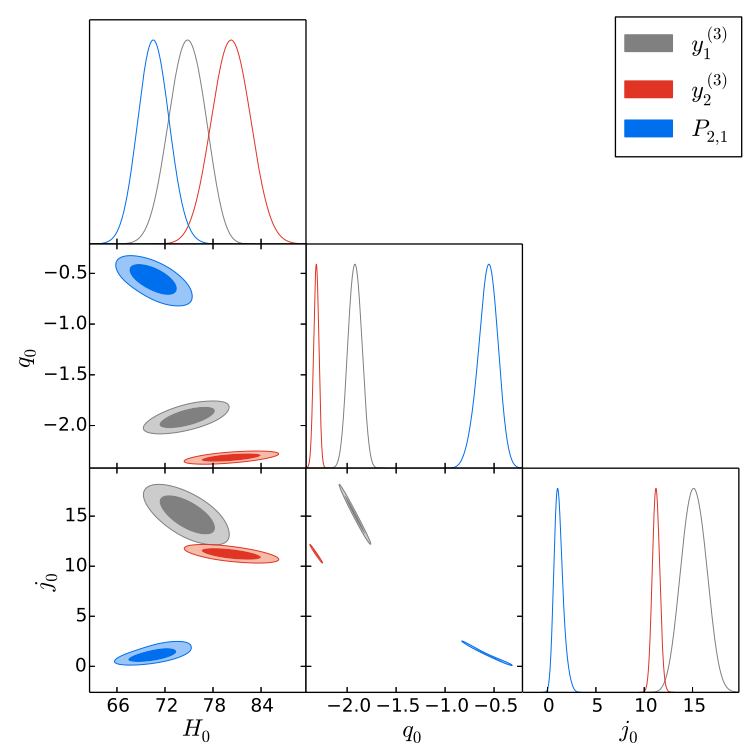

(a) Third-order analyses

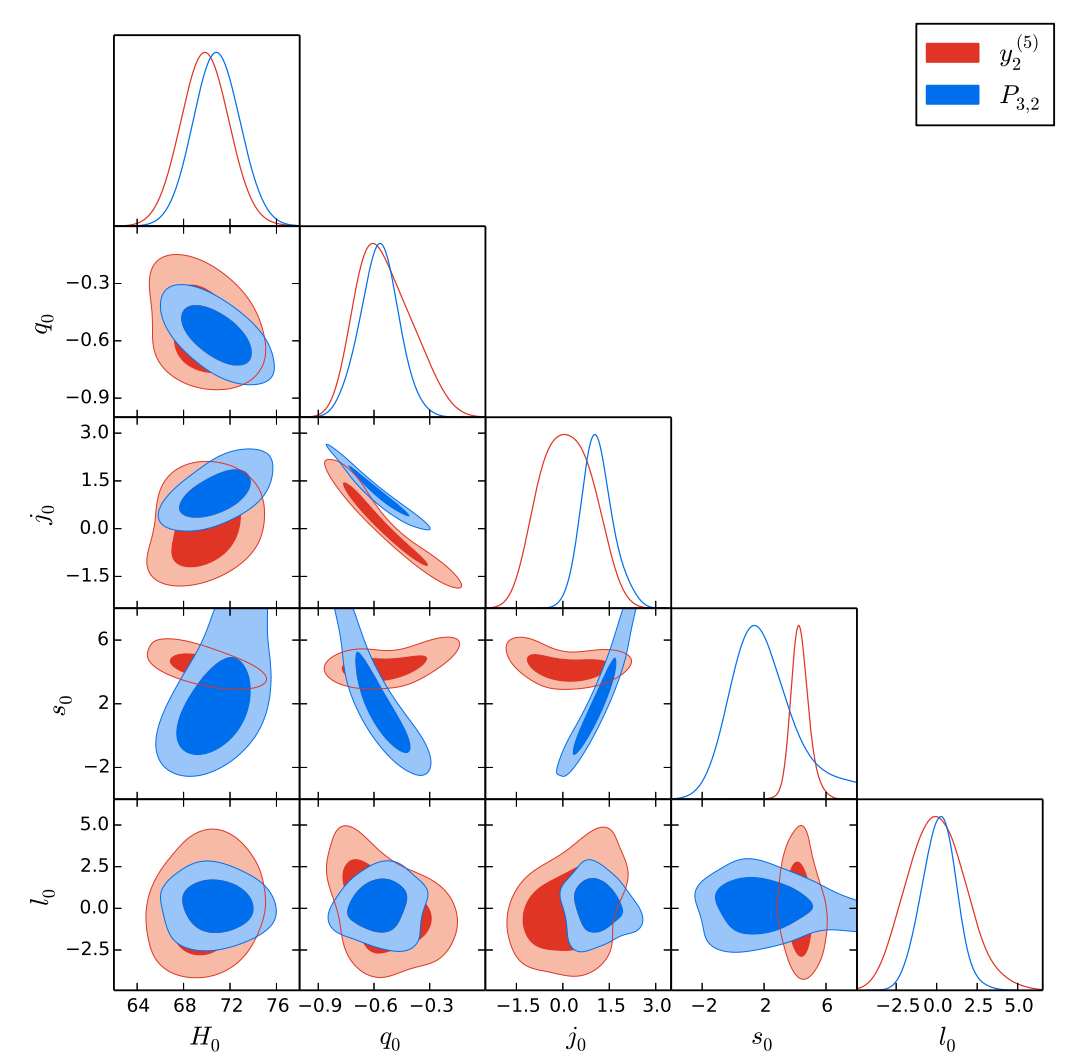

(c) Fifth-order analyses

(c) Eifth-order analyses

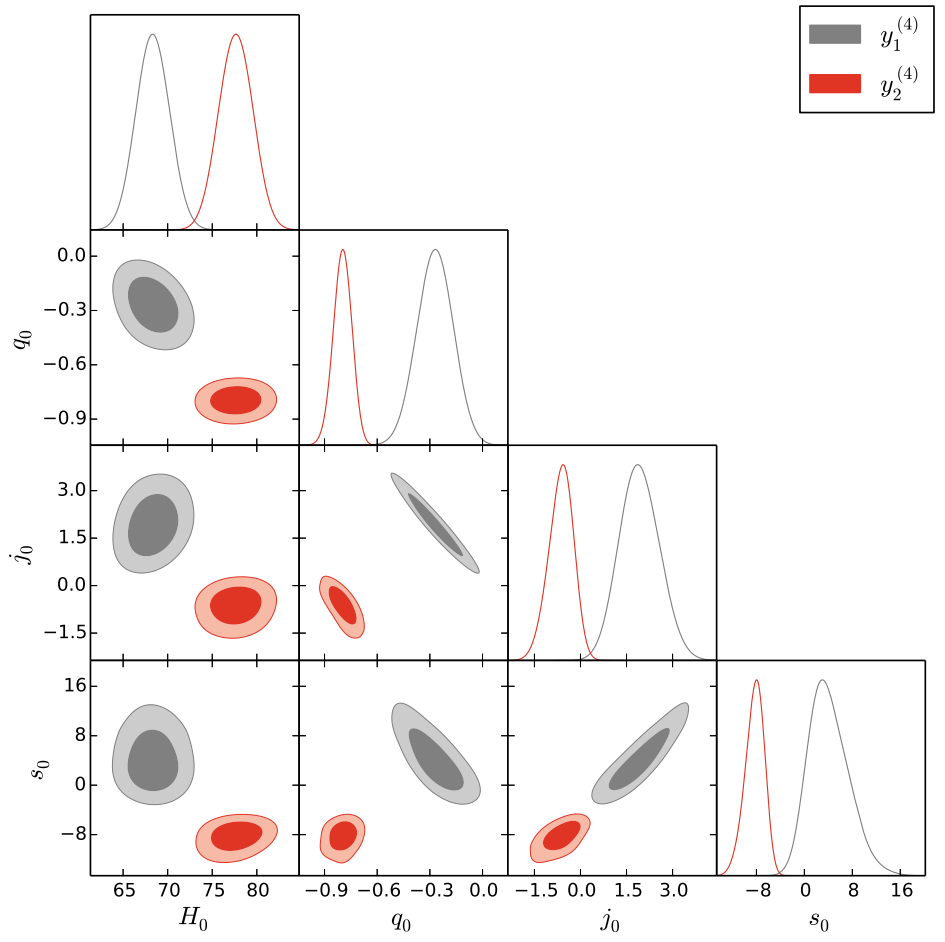

(b) Fourth-order analyses

Figure 3. Combined low and high-redshift $(\mathrm{SNe} \mathrm{Ia}+\mathrm{OHD}+\mathcal{R})$ constraints on the cosmographic parameters at the $68 \%$ and $95 \%$ C.L. for different techniques. 
$y$-variables are many $\sigma \mathrm{s}$ away from the predictions of the $\Lambda$ CDM model, whereas the constraints of the $(2,1)$ Padé polynomial are fully compatible with those of $\Lambda \mathrm{CDM}$ (see top-left panel of Figure 3). These results are confirmed by the AIC and BIC values, which indicate very strong evidence against the $y$-variables (cf. Table 2). At the fourth-order expansion, the $y$-variables give results in $\sim 3 \sigma$ tension with each other (see top-right panel of Figure 3), and values of the set $\left(q_{0}, s_{0}, l_{0}\right)$ are, in both cases, $\gtrsim 2 \sigma$ away from those predicted by the $\Lambda$ CDM model (cf. Table 2). On the other hand, the $(2,2)$ Padé rational polynomial does not represent a suitable approximation of $d_{L}(z)$ in the high-redshift domains, where it shows a plateau behaviour (see Figure 1) due to the presence of the same powers of $z$ in the numerator and denominator. At the fifth-order expansion, the cosmographic series, provided by the $y_{1}$-variable, is not constrained by the data, while the $y_{2}$-variable is characterized by the lowest $\mathrm{AIC}$ and BIC values among the different techniques of the same order (cf. Table 2). The results of the $(3,2)$ Padé polynomial are in agreement with the predictions of the $\Lambda \mathrm{CDM}$ model at the $68 \%$ C.L., although the large uncertainties on the high-order cosmographic parameters $s_{0}$ and $l_{0}$ (see bottom panel of Figure 3). See also Lusso et al. (2019) for a discussion on $\Lambda \mathrm{CDM}$ tension.

The previous considerations suggest that modelindependent geometrical tests could be relevant to check the goodness of our findings. In particular, they are essential to understand if the concordance paradigm is effectively recovered in the picture of our numerical results. To this end, the use of statefinder diagnostic is of utmost importance because these quantities depend on space-time geometry only ${ }^{7}$ (Shafieloo, Sahni \& Starobinsky 2012). The statefinder prescription will be used in the next subsection, motivated by the fact that, at both 1 and $2 \sigma$ confidence levels, using low and high redshift data, the $\Lambda \mathrm{CDM}$ predictions are not completely fulfilled by our analyses. For example, the most stable techniques, i.e. the auxiliary variables and Padé polynomials that minimize the $\mathrm{AIC}$ and BIC values, suggest that the jerk parameter seems to be slightly larger than the one predicted by the concordance paradigm, i.e. $j_{0}=1$. We discuss in detail this result in the next subsection.

To summarize, the $y_{2}$-parametrization performs relatively better than the $y_{1}$-parametrization over the whole redshift domain, while the most accurate and stable approximations of $d_{L}(z)$, at the high-redshift regimes, are given by the Padé technique. Given the low number of degrees of freedom and its ability to properly constrain the cosmographic series, the $(2,1)$ Padé approximation is the best polynomial to be used in high-redshift cosmography. The full list of results is summarized in Table 3.

\subsection{Discussion on a possible dark energy evolution}

In the previous subsection, we left open the possibility that dark energy could slightly evolve with time. This is plausible at both $1 \sigma$ and $2 \sigma$ confidence levels on the basis of our numerical outcomes. Let us now investigate better this fact

7 This is a consequence of the fact that they are functions of Hubble rate or scale factor. through the use of the well established statefinder diagnostics. In particular the statefinder parameter is defined by

$O m h^{2}\left(z_{i} ; z_{j}\right)=\frac{h^{2}\left(z_{i}\right)-h^{2}\left(z_{j}\right)}{\left(1+z_{i}\right)^{3}-\left(1+z_{j}\right)^{3}}$,

where $h(z)=H(z) / 100 \mathrm{~km} / \mathrm{sec} / \mathrm{Mpc}$ and $z_{i}, z_{j}$ represent a set of two redshifts at which the Hubble rates of Eq. (34) are computed. Concerning the concordance paradigm, one immediately has $O m h^{2}=\Omega_{m 0} h^{2}$ and looking at the most recent Planck constraint, we can immediately deduce that

$O m h^{2}=\Omega_{m 0} h^{2}=0.14240 \pm 0.00087$.

This value is in tension with the $\Lambda$ CDM-based prediction, stable around $\Omega_{m 0} h^{2} \simeq 0.12$, when Baryonic Acoustic Oscillation measurements are considered (see for details Sahni, Shafieloo \& Starobinsky (2014); Delubac et al. (2015)).

To go further and checking this with cosmography, it is possible to reproduce Eq. (35), starting with the recipe that a generic dark energy model evolves as $H(z)=$ $H_{0} \sqrt{\Omega_{m 0}(1+z)^{3}+\Omega_{D E 0} G(z)}$, where $G(z)$ satisfies $G(z) \rightarrow$ 1 , as $z=0$, with $\Omega_{D E 0} \equiv 1-\Omega_{m 0}$. Thus, we can write

$q(z)=-1+\frac{(1+z)\left[3 \Omega_{m 0}(1+z)^{2}+\Omega_{D E 0} G^{\prime}(z)\right]}{2\left[\Omega_{m 0}(1+z)^{3}+\Omega_{D E 0} G(z)\right]}$,

that, for a genuine cosmological constant contribution, becomes in terms of cosmography:

$O m h^{2}=\frac{2}{3}\left(q_{0}+1\right) h^{2}$.

Taking our numerics, it is immediately possible to find the statefinder values at small and high redshift domains by involving $h=h_{0}$ and $q_{0}$ as reported in Tabs. I and II. Thus, at small redshifts, i.e. using only SNe Ia and OHD data sets, we have

$$
\begin{aligned}
O m h_{y, \text { small }}^{2} & =0.1316 \pm 0.0618(0.1188), \\
O m h_{P, \text { small }}^{2} & =0.0864 \pm 0.0466(0.0928),
\end{aligned}
$$

in which we evaluated, through Eq. (37), the best auxiliary variable approximation, i.e. $y_{2}^{(4)}$, and the optimal rational approximation, i.e. $P_{21}$, respectively.

The same procedure can be considered at higher redshifts, where we have instead

$$
\begin{aligned}
O m h_{y, h i g h}^{2} & =0.1498 \pm 0.0599(0.1100), \\
O m h_{P, h i g h}^{2} & =0.1441 \pm 0.0416(0.0860)
\end{aligned}
$$

for the most suitable auxiliary variable, this time $y_{2}^{(5)}$, and the optimal version of Padé approximation, given now by $P_{3,2}$, respectively. We explored only these four scenarios, because these approximations are the statistically favorite ones, as confirmed by AIC and BIC statistical criteria, as one can see in Tabs. I and II.

At small redshifts only, the tensions among our predictions and the Planck measurements still persist, becoming larger in the rational approximation case, i.e. for Padé $(2,1)$. This can be interpreted as previously stated throughout the manuscript: the use of rational approximations works better only when the redshift is large and becomes unnecessary at large scales, i.e. when the redshift becomes smaller. At larger redshifts, however, adding the $\mathcal{R}$ measure, the tension between our $\Lambda \mathrm{CDM}$ predictions and the Planck results, 
Cosmographic technique Low redshifts

High redshifts

Taylor $\$ The series is suitable when data are inside $z<1$.

- Good agreement with the $\Lambda \mathrm{CDM}$ model, up to the fourth order.

- Limited to short intervals of data.
High-redshift measurements are non-predictive.

- Numerical outcomes are both nonphysical and non-convergent.

- Useless for making physical predictions.

\begin{tabular}{|c|c|c|}
\hline$y$-variable & $\begin{array}{l}\text { Third-order } y_{1} \text { is unsuitable as } \\
\text { more than } 2 \sigma \text { away from Taylor cos- } \\
\text { mography. } \\
\text { At the fourth order, } y_{2} \text { works } \\
\text { much better than } y_{1} \text {. } \\
\text { No apparent need to adopt } y \text { - } \\
\text { variables instead of Taylor series. }\end{array}$ & $\begin{array}{l}\text { At all orders, } y_{1} \text { is unsuitable. } \\
\text { Only the fifth-order } y_{2} \text { is suitable } \\
\text { and shows the lowest } \mathrm{AIC} \text { and BIC val- } \\
\text { ues among all the fits. } \\
\Delta \mathrm{AIC} \text { and } \Delta \mathrm{BIC} \text { indicate } \\
\text { very strong evidence against the } \\
y \text {-variables, except for the fifth-order } \\
y_{2} \text {. }\end{array}$ \\
\hline Padé & $\begin{array}{l}\text { The }(2,1) \text { polynomial is statisti- } \\
\text { cally the best-performing approxima- } \\
\text { tion. } \\
\text { give similar results, but with unsuit- } \\
\text { able errors. } \\
\text { No apparent need to use Padé se- } \\
\text { ries of any orders over the Taylor series. }\end{array}$ & $\begin{array}{l}\text { The }(2,2) \text { Padé polynomial is } \\
\text { strongly disfavoured and should be } \\
\text { ruled out. } \\
\text { The }(2,1) \text { and }(3,2) \text { polynomials } \\
\text { are suitable approximations, but with } \\
\text { very large uncertainties. } \\
\text { The }(2,1) \text { polynomial performs } \\
\text { better than the } y \text {-variables. }\end{array}$ \\
\hline
\end{tabular}

Table 3. Comparison among the different cosmographic techniques.

is alleviated. As a consequence, the $\Lambda \mathrm{CDM}$ model can be recovered somehow, albeit in both cases the error bars are quite large at $1 \sigma$ confidence level yet, leaving open the possibility that $O m h^{2}$ persists being in tension ${ }^{8}$. So, even in this case a dark energy evolution is not excluded $a$ priori. To justify our prescriptions, one can imagine that possible systematics can affect the whole analysis in general, without substantially alleviating the tension presented in Delubac et al. (2015). This limitation has been discussed for example in Busti et al. (2015).

In addition, for generic dark energy models, inverting Eq. (36), cosmography suggests that

$\Omega_{m 0}=\frac{G_{0}^{\prime}-2\left(q_{0}+1\right)}{G_{0}^{\prime}-3}$.

Moreover, by virtue of the dark energy form, one immediately finds

$\frac{G_{0}^{\prime \prime}}{3-G_{0}^{\prime}} \geqslant \frac{2 G_{0}^{\prime}}{3-G_{0}^{\prime}}$

which has been evaluated assuming $j_{0} \geqslant 1$, as our numerical results partially seem to indicate, and conventionally considering that $G^{\prime}(0) \equiv G_{0}^{\prime}$ and $G^{\prime \prime}(0) \equiv G_{0}^{\prime \prime}$. From the above considerations, cosmographic departures of $O m h^{2}$, above depicted, can represent a signal that $\Lambda$ does not drive the

8 For the sake of completeness, we notice that we computed our error bars through a standard logarithmic formula. As errors over $q_{0}$ and $h_{0}$, we considered the mean values among upper and lower error limits. universe to accelerate today ${ }^{9}$. This would imply that some sort of modifications of Einstein's gravity is still possible. By looking at Eqs. (42) and (43), models in which dark energy, and in particular the cosmological constant, is screened (or compensated) by a dynamically evolving counter-term are permitted, once $G_{0}^{\prime} \neq 3$, choosing the sign of both $G_{0}^{\prime}$ and $G_{0}^{\prime \prime}$. Concluding, the dark energy evolution is not excluded $a$ priori through the here-developed cosmographic methods. A possible tension among $\Omega_{m 0} h^{2}$ measured by Planck and expected by cosmography can lead to the need of new physics. A more accurate study on these aspects will be the object of future efforts toward the refinements of a new high-redshift cosmography. This would characterize the signs of $G_{0}^{\prime}$ and $G_{0}^{\prime \prime}$ with increasing accuracy, disclosing at small and large redshifts whether dark energy evolves with time.

\section{FINAL OUTLOOKS AND PERSPECTIVES}

We critically revised the role of cosmographic treatments and, in particular, the reconstruction techniques widely adopted in the literature. The main issues of cosmography have been considered focusing on the basic demands of the convergence problem, related to series expansions around $z=0$, while cosmic data go further this limit. We first reviewed the two main scenarios introduced to heal the convergence issue, i.e. the rational approximations and the auxil-

9 We note that the denominator sign of Eq. (43) is not specified a priori. Thence, if positive, one has $G_{0}^{\prime \prime}>2 G_{0}^{\prime}$, otherwise $G_{0}^{\prime \prime}<$ $-2\left|G_{0}^{\prime}\right|$. 
iary variables, showing how to construct the Padé and the $y$ variable cosmography, respectively. From a theoretical point of view, we developed the main rules and the basic requirements which any reconstruction should have. An important result is that lower order might be favored than higher ones. The theoretical issues associated with the use of $y$-variables have been also discussed. We took into account a hierarchy between cosmographic coefficients, starting from the thirdorder expansion up to the fifth one.

Although appealing, $y$-variable cosmography becomes non predictive already from a theoretical point of view. Thus, in agreement with previous approaches, we showed that parametrizations of the redshift variables and alternatives to Taylor expansions are disfavoured if used arbitrarily, i.e. without calibrating the orders with cosmic data. Specifically, we considered the two parametrizations $y_{1}=1-a$ and $y_{2}=\arctan \left(a^{-1}-1\right)$, and some Padé expansions. It is worth noticing that approximations different from $y_{1}$ and $y_{2}$, diverging for $z \rightarrow \infty$, are not theoretically viable to heal the convergence issue. Afterwards, we focused on the $(1,1)$, $(2,1),(2,2),(3,2)$ and $(4,1)$ Padé approximations, discarding other orders which show bad behaviours if compared to the fiducial $\Lambda \mathrm{CDM}$ model. We therefore provided numerical fits by means of Monte Carlo integration of combined Supernova Ia data and Hubble measurements. Specifically, we adopted the most recent Pantheon data set, free from nuisance parameters due to the adopted condition of a spatially flat universe, and the observational Hubble data acquired through the differential age technique. We tested different cosmographic orders by splitting our study at late times, and early times through the use of the CMB shift parameter. As theoretically argued, we found non conclusive or non convergent results in several cases of interest, erroneously used in the literature. Moreover, the AIC and BIC selection criteria have been adopted as tools for inferring the statistical significance of a given scenario with respect to the reference $\Lambda \mathrm{CDM}$ model.

In particular, in the low-redshift regime, we noted that the results of the third-order $y_{1}$-variable are substantially different than $2 \sigma$ with respect to the corresponding Taylor constraints, whereas the $(2,1)$ Padé polynomial turns out to be the best-performing third-order approximation. Going further with the orders, still the $y_{2}$-variable behaves better than the $y_{1}$-variable, while a strong statistical evidence against the $(2,2)$ Padé polynomial is found. At the fifth-order expansion, large uncertainties occur, making cosmography non predictive, as expected. The value of $l_{0}$ results to be unbounded in every analysis, and a statistical significance seems to favor the $(2,1)$ Padé polynomial over the $(3,2)$ one, although the interesting results provided by the latter.

Involving high-redshift measurements, we found that every order of Taylor polynomials fails to be predictive, as theoretically expected. Even the $y$-variables are many $\sigma \mathrm{s}$ away from the predictions of the $\Lambda \mathrm{CDM}$ model, whereas the constraints of the $(2,1)$ Padé polynomial are fully compatible with those ones. Increasing the order leads to more problematic results, as the fourth-order $y$-variables give results that are in $\sim 3 \sigma$ tension with each other, and the set $\left(q_{0}, s_{0}, l_{0}\right)$ is, in both cases, in $\gtrsim 2 \sigma$ tension with the predictions of the $\Lambda$ CDM model. The $(2,2)$ Padé rational polynomial does not represent a suitable approximation of $d_{L}(z)$ at high redshifts, due to the presence of the same powers of $z$ in the numerator and denominator. The main troubles occur as soon as the fifth-order expansions are involved. The cosmographic series provided by the $y_{1}$-variable is not constrained by the data, while we found that the $y_{2}$ variable works better than any other expansions. Our results show that the $y_{2}$-parametrization performs relatively better than the $y_{1}$-parametrization over the whole redshift domains, while the most accurate and stable approximations of $d_{L}(z)$ at the high-redshift regimes are due to the Padé approximations. The Bayesian information criteria suggest that the $(2,1)$ Padé approximation is optimal, also due to the low number of variables involved in the approximations. A tension with the concordance paradigm was also found by investigating the statefinder diagnostics. In particular, comparing our results with the ones got by Planck, we showed that the $\Lambda \mathrm{CDM}$ model seems to predict smaller values of $O m h^{2}$, in net tension with Planck results. This is particularly true at small redshifts, whereas by adding the $\mathcal{R}$ measure, at smaller scales and higher redshifts, it is weakly alleviated, albeit error bars do not permit to conclude that the tension is removed. Indeed, it seems that both cases leave open the possibility that dark energy evolves in time.

Future works will focus on how to generalize the aforementioned results by also considering alternative rational approximations. We will focus on how to reduce systematics, minimizing the expansion orders and maximizing the quality of numerical outcomes up to the $l_{0}$ order. The same procedure, reported here, will be considered in view of the whole Planck data to check whether cosmography is able to discriminate the evolution of dark energy from the cosmological constant evolution.

As a final remark, it is worth saying that the cosmographic approach can provide a useful feedback on the behaviour of dark energy, in particular at large redshifts of the order $z \sim 10$. This is valuable in order to see how the latetime matter domination exploding (oscillating) terms (for example in modified gravity) may (or may not) affect the equation of state of dark energy. This will be the topic of a forthcoming paper.

\section{ACKNOWLEDGEMENTS}

The authors acknowledge the support of INFN (iniziative specifiche QGSKY and MoonLIGHT-2). This paper is partially based upon work from COST action CA15117 (CANTATA), supported by COST (European Cooperation in Science and Technology). O.L. acknowledges the support provided by the Ministry of Education and Science of the Republic of Kazakhstan, Program IRN: BR05236494.

\section{References}

Ade P.A.R. et al. (Planck Collaboration), 2016, A\&A, 594, A14 Aghanim N. et al. (Planck Collaboration), 2018, preprint (arXiv:1807.06209)

Akaike H., 1974, IEEE T. Automat. Contr., 19, 716

Akrami Y. et al. (Planck Collaboration), 2018, preprint (arXiv:1807.06211)

Amendola L., Tsujikawa S., 2015, Dark Energy: Theory and Observations. Cambridge Univ. Press, Cambridge

Aviles A., Cervantes-Cota J. L., 2011, Phys. Rev. D, 84, 083515 
Aviles A., Gruber C., Luongo O., Quevedo H., 2012, Phys. Rev. $\mathrm{D}, 86,123516$

Aviles A., Bravetti A., Capozziello S., Luongo O., 2013, Phys. Rev. D, 87, 044012

Aviles A., Bravetti A., Capozziello S., Luongo O., 2014, Phys. Rev. D, 90, 043531

Bamba K., Capozziello S., Nojiri S., Odintsov S. D., 2012, Ap\&SS, 342,155

Benetti M., Capozziello S., 2019, J. Cosm. Astrop. Phys., 1912, 008

Bonilla A., D'Agostino R., Nunes R. C., de Araujo J. C. N., 2020, J. Cosm. Astrop. Phys., 2003, 015

Busti, V. C., Holanda R. F. L., Clarkson C., 2013, J. Cosm. Astrop. Phys., 11, 020

Busti V. C., Dunsby P. K. S., de la Cruz-Dombriz A., Saez-Gomez D., 2015, Phys. Rev. D, 92, 123512

Escamilla-Rivera C., Capozziello S., 2019, Int. J. Mod. Phys. D, 28,1950154

Escamilla-Rivera C., Quintero M. A. C., Capozziello S., 2020, J. Cosm. Astrop. Phys., 2003, 008

Capozziello S., 2002, Int. J. Mod. Phys. D, 11, 483

Capozziello S., D'Agostino R., Luongo O., 2018, MNRAS, 476, 3924

Capozziello S., D’Agostino R., Luongo O., 2018, J. Cosm. Astrop. Phys., 008, 1805

Capozziello S., Ruchika, Sen A. A., 2019, MNRAS, 484, 4484

Capozziello S., D'Agostino R., Luongo O., 2019, Int. J. Mod. Phys. D, 28, 1930016

Carroll S. M., 2001, Living Rev. Rel., 4, 1

Cattoen C., Visser M., 2007, Class. Quant. Grav., 24, 5985

Cattoen C., Visser M., 2008, Phys. Rev. D, 78, 063501

Chevallier M., Polarski D., 2001, Int. J. Mod. Phys. D. 10, 213224

Clarkson C., Bassett B., Lu T. H. C., 2008, Phys. Rev. Lett., 101, 011301

Clifton T., Ferreira P. G., Padilla A., Skordis C., 2012, Phys. Rept., 513, 1

Copeland E. J., Sami M., Tsujikawa S., 2006, Int. J. Mod. Phys. $\mathrm{D}, 15,1753$

D'Agostino R., 2019, Phys. Rev. D, 99, 103524

D'Agostino R., Nunes R. C., 2019, Phys. Rev. D, 100, 044041

Delubac, T. et al., 2015, A\&A, 574, A59

Di Valentino E., Melchiorri A., Silk J., 2020, Nature Astron., 4, 196

Dunsby P. K. S., Luongo O., 2016, Int. J. Geom. Meth. Mod. Phys., 13, 1630002

Dunsby P. K. S., Luongo O., Reverberi L., 2016, Phys. Rev. D, 94, 083525

Efstathiou G., Bond J., 1999, MNRAS, 304, 75

Farooq O., Ratra B., 2013, Phys. Lett. B, 723, 1

Gruber C., Luongo O., 2014, Phys. Rev. D, 89, 103506

Krantz S. G., Parks, H. R., 1992, A primer of real analytic functions. Birkhauser

Kunz M., Trotta R., Parkinson D., 2006, Phys. Rev. D, 74, 023503

Harrison E. R., 1976, Nature, 260, 591

Jimenez R., Loeb A., 2002, ApJ, 573, 37

Linder E. V., 2003, Phys. Rev. Lett., 90, 091301

Lobo F. S. N., Mimoso J. P., Visser M., 2020, preprint (arXiv:2001.11964)

Luongo O., 2011, Mod. Phys. Lett. A, 26, 1459

Luongo O., 2013, Mod. Phys. Lett. A, 28, 1350080

Luongo O., Muccino M., 2018, Phys. Rev. D, 98, 103520

Luongo O., Quevedo H., 2014, Int. J. Mod. Phys. D, 23, 1450012

Luongo O., Quevedo H., 2014, Gen. Rel. Grav., 46, 1649

Lusso E., Piedipalumbo E., Risaliti G., Paolillo M., Bisogni S., Nardini E., Amati L., 2019, A\&A, 628, L4

Maartens R., Majerotto E., 2006, Phys. Rev. D, 74, 023004

Nesseris S., Shafieloo A., 2010, MNRAS, 408, 1879
Nojiri S., Odintsov S. D., 2007, Int. J. Geom. Meth. Mod. Phys., 4,115

Nojiri S., Odintsov S. D., Oikonomou V. K., 2017, Phys. Rept., 692,1

Padmanabhan T., 2010, Rept. Prog. Phys., 73, 046901

Peebles P. J. E., Ratra B., 2003, Rev. Mod. Phys., 75, 559

Piedipalumbo E., Della Moglie E., De Laurentis M., Scudellaro P., 2014, MNRAS, 441, 3643

Risaliti G., Lusso E., 2015, ApJ, 815, 33

Risaliti G., Lusso E., 2019, Nature Astron., 3, 272

Sahni V., Starobinsky A., 2000, Int. J. Mod. Phys. D, 9, 373

Sahni V., Shafieloo A., Starobinsky A. A., 2008, Phys. Rev. D, 78,103502

Sahni V., Shafieloo A., Starobinsky A. A., 2014, ApJ, 793, L40

Schwarz G., 1978, Ann. Stat., 6, 461

Scolnic D. M. et al., 2018, ApJ, 859, 101

Shafieloo A., Sahni V., Starobinsky A. A., 2012, Phys. Rev. D, 86,103527

Starobinsky A. A., 1980, Phys. Lett. B, 91, 99

Tsujikawa S., 2010, Dark energy: investigation and modeling, preprint (arXiv:1004.1493)

Uzan J. P., Clarkson C., Ellis G. F. R., 2008, Phys. Rev. Lett. 100,191303

Visser M., 2005, Gen. Rel. Grav., 37, 1541

Visser M., 2015, Class. Quant. Grav., 32, 135007

Wei H., Yan X. P., Zhou Y. N., J. Cosm. Astrop. Phys., 1401, 045 (2014).

Weinberg S., 1972, Gravitation and Cosmology. Wiley, New York Weinberg S., 1989, Rev. Mod. Phys., 61, 1

Zunckel C., Clarkson C., 2008, Phys. Rev. Lett., 101, 181301 


\section{APPENDIX}

Here, all the cosmographic expansions, up to the fifth order, which we adopted in this study are reported.

- Taylor approximations of the luminosity distance and the Hubble rate:

$$
\begin{aligned}
d_{L}(z)= & \frac{1}{H_{0}}\left[z+\frac{1}{2}\left(1-q_{0}\right) z^{2}-\frac{1}{6}\left(1-q_{0}-3 q_{0}^{2}+j_{0}\right) z^{3}+\frac{1}{24}\left(2-2 q_{0}-15 q_{0}^{2}-15 q_{0}^{3}+5 j_{0}\right.\right. \\
& \left.+10 q_{0} j_{0}+s_{0}\right) z^{4}+\left(-\frac{1}{20}-\frac{9 j_{0}}{40}+\frac{j_{0}^{2}}{12}-\frac{l_{0}}{120}+\frac{q_{0}}{20}-\frac{11 j_{0} q_{0}}{12}+\frac{27 q_{0}^{2}}{40}-\frac{7 j_{0} q_{0}^{2}}{8}\right. \\
& \left.\left.+\frac{11 q_{0}^{3}}{8}+\frac{7 q_{0}^{4}}{8}-\frac{11 s_{0}}{120}-\frac{q_{0} s_{0}}{8}\right) z^{5}\right] \\
H(z)= & H_{0}\left[1+\left(1+q_{0}\right) z+\frac{1}{2}\left(j_{0}-q_{0}^{2}\right) z^{2}-\frac{1}{6}\left(-3 q_{0}^{2}-3 q_{0}^{3}+j_{0}\left(3+4 q_{0}\right)+s_{0}\right) z^{3}\right. \\
& \left.+\frac{1}{24}\left(-4 j_{0}^{2}+l_{0}-12 q_{0}^{2}-24 q_{0}^{3}-15 q_{0}^{4}+j_{0}\left(12+32 q_{0}+25 q_{0}^{2}\right)+8 s_{0}+7 q_{0} s_{0}\right) z^{4}\right] .
\end{aligned}
$$

- $y_{1}$-variable approximations of the luminosity distance and the Hubble rate:

$$
\begin{aligned}
d_{L}\left(y_{1}\right)= & \frac{1}{H_{0}}\left[y_{1}+\frac{1}{2}\left(3-q_{0}\right) y_{1}^{2}+\frac{1}{6}\left(11-j_{0}-5 q_{0}+3 q_{0}^{2}\right) y_{1}^{3}+\frac{1}{24}\left(50-7 j_{0}-26 q_{0}+10 j_{0} q_{0}\right.\right. \\
& \left.+21 q_{0}^{2}-15 q_{0}^{3}+s_{0}\right) y_{1}^{4}+\frac{1}{120}\left(274-47 j_{0}+10 j_{0}^{2}-l_{0}-154 q_{0}+90 j_{0} q_{0}+141 q_{0}^{2}\right. \\
& \left.\left.-105 j_{0} q_{0}^{2}-135 q_{0}^{3}+105 q_{0}^{4}+9 s_{0}-15 q_{0} s_{0}\right) y_{1}^{5}\right], \\
H\left(y_{1}\right)= & H_{0}\left[1+\left(1+q_{0}\right) y_{1}+\frac{1}{2}\left(2+j_{0}+2 q_{0}-q_{0}^{2}\right) y_{1}^{2}-\frac{1}{6}\left(-6-6 q_{0}+3 q_{0}^{2}-3 q_{0}^{3}\right.\right. \\
& \left.+j_{0}\left(-3+4 q_{0}\right)+s_{0}\right) y_{1}^{3}-\frac{1}{24}\left(-24+4 j_{0}^{2}-l_{0}-24 q_{0}+12 q_{0}^{2}-12 q_{0}^{3}+15 q_{0}^{4}\right. \\
& \left.\left.+j_{0}\left(-12+16 q_{0}-25 q_{0}^{2}\right)+4 s_{0}-7 q_{0} s_{0}\right) y_{1}^{4}\right] .
\end{aligned}
$$

- $y_{2}$-variable approximations of the luminosity distance and the Hubble rate:

$$
\begin{aligned}
d_{L}\left(y_{2}\right)= & \frac{1}{H_{0}}\left[y_{2}+\frac{1}{2}\left(1-q_{0}\right) y_{2}^{2}+\frac{1}{6}\left(1-j_{0}+q_{0}+3 q_{0}^{2}\right) y_{2}^{3}+\frac{1}{24}\left(10+5 j_{0}-10 q_{0}+10 j_{0} q_{0}\right.\right. \\
& \left.-15 q_{0}^{2}-15 q_{0}^{3}+s_{0}\right) y_{2}^{4}+\frac{1}{120}\left(-10-47 j_{0}+10 j_{0}^{2}-l_{0}+26 q_{0}-110 j_{0} q_{0}+141 q_{0}^{2}\right. \\
& \left.\left.-105 j_{0} q_{0}^{2}+165 q_{0}^{3}+105 q_{0}^{4}-11 s_{0}-15 q_{0} s_{0}\right) y_{2}^{5}\right], \\
H\left(y_{2}\right)= & H_{0}\left[1+\left(1+q_{0}\right) y_{2}+\frac{1}{2}\left(j_{0}-q_{0}^{2}\right) y_{2}^{2}+\frac{1}{6}\left(2+2 q_{0}+3 q_{0}^{2}+3 q_{0}^{3}-j_{0}\left(3+4 q_{0}\right)-s_{0}\right) y_{2}^{3}\right. \\
& \left.+\frac{1}{24}\left(-4 j_{0}^{2}+l_{0}-20 q_{0}^{2}-24 q_{0}^{3}-15 q_{0}^{4}+j_{0}\left(20+32 q_{0}+25 q_{0}^{2}\right)+8 s_{0}+7 q_{0} s_{0}\right) y_{2}^{4}\right] .
\end{aligned}
$$

- $(2,1)$ Padé approximation of the luminosity distance:

$P_{2,1}(z)=\frac{1}{H_{0}}\left[\frac{z\left(6\left(-1+q_{0}\right)+\left(-5-2 j_{0}+q_{0}\left(8+3 q_{0}\right)\right) z\right)}{-2\left(3+z+j_{0} z\right)+2 q_{0}\left(3+z+3 q_{0} z\right)}\right]$.

- $(2,2)$ Padé approximation of the luminosity distance:

$$
\begin{aligned}
P_{2,2}(z) & =\frac{1}{H_{0}}\left(6 z\left(10+9 z-6 q_{0}^{3} z+s_{0} z-2 q_{0}^{2}(3+7 z)-q_{0}(16+19 z)+j_{0}\left(4+\left(9+6 q_{0}\right) z\right)\right) /\right. \\
& \left(60+24 z+6 s_{0} z-2 z^{2}+4 j_{0}^{2} z^{2}-9 q_{0}^{4} z^{2}-3 s_{0} z^{2}+6 q_{0}^{3} z(-9+4 z)+q_{0}^{2}(-36-114 z\right. \\
& \left.\left.+19 z^{2}\right)+j_{0}\left(24+6\left(7+8 q_{0}\right) z+\left(-7-23 q_{0}+6 q_{0}^{2}\right) z^{2}\right)+q_{0}\left(-96-36 z+\left(4+3 s_{0}\right) z^{2}\right)\right) .
\end{aligned}
$$


- $(3,2)$ Padé approximation of the luminosity distance:

$$
\begin{aligned}
P_{3,2}(z) & =\frac{1}{H_{0}}\left(z \left(-120-180 s_{0}-156 z-36 l_{0} z-426 s_{0} z-40 z^{2}+80 j_{0}^{3} z^{2}-30 l_{0} z^{2}-135 q_{0}^{6} z^{2}\right.\right. \\
& -210 s_{0} z^{2}+15 s_{0}^{2} z^{2}-270 q_{0}^{5} z(3+4 z)+9 q_{0}^{4}\left(-60+50 z+63 z^{2}\right)+2 q_{0}^{3}(720+1767 z \\
& \left.+887 z^{2}\right)+3 j_{0}^{2}\left(80+20\left(13+2 q_{0}\right) z+\left(177+40 q_{0}-60 q_{0}^{2}\right) z^{2}\right)+6 q_{0}^{2}\left(190+5\left(67+9 s_{0}\right) z\right. \\
& \left.+\left(125+3 l_{0}+58 s_{0}\right) z^{2}\right)-6 q_{0}\left(s_{0}\left(-30+4 z+17 z^{2}\right)-2\left(20+\left(31+3 l_{0}\right) z+\left(9+4 l_{0}\right) z^{2}\right)\right) \\
& +6 j_{0}\left(-70+\left(-127+10 s_{0}\right) z+45 q_{0}^{4} z^{2}+\left(-47-2 l_{0}+13 s_{0}\right) z^{2}+5 q_{0}^{3} z(30+41 z)\right. \\
& \left.\left.\left.-3 q_{0}^{2}\left(-20+75 z+69 z^{2}\right)+2 q_{0}\left(-115-274 z+\left(-136+5 s_{0}\right) z^{2}\right)\right)\right)\right) /\left(3 \left(-40-60 s_{0}\right.\right. \\
& -32 z-12 l_{0} z-112 s_{0} z-4 z^{2}+40 j_{0}^{3} z^{2}-4 l_{0} z^{2}-135 q_{0}^{6} z^{2}-24 s_{0} z^{2}+5 s_{0}^{2} z^{2} \\
& -30 q_{0}^{5} z(12+5 z)+3 q_{0}^{4}\left(-60+160 z+71 z^{2}\right)+j_{0}^{2}\left(80+20\left(11+4 q_{0}\right) z+\left(57+20 q_{0}\right.\right. \\
& \left.\left.-40 q_{0}^{2}\right) z^{2}\right)+6 q_{0}^{3}\left(80+188 z+\left(44+5 s_{0}\right) z^{2}\right)+2 q_{0}^{2}\left(190+20\left(13+3 s_{0}\right) z+\left(46+6 l_{0}\right.\right. \\
& \left.\left.+21 s_{0}\right) z^{2}\right)+4 q_{0}\left(20+\left(16+3 l_{0}\right) z+\left(2+l_{0}\right) z^{2}+s_{0}\left(15-17 z-9 z^{2}\right)\right)+2 j_{0}(-70 \\
& +2\left(-46+5 s_{0}\right) z+90 q_{0}^{4} z^{2}+\left(-16-2 l_{0}+3 s_{0}\right) z^{2}+15 q_{0}^{3} z(12+5 z)+q_{0}^{2}(60-370 z \\
& \left.\left.\left.\left.-141 z^{2}\right)+2 q_{0}\left(-115-234 z+2\left(-26+5 s_{0}\right) z^{2}\right)\right)\right)\right) .
\end{aligned}
$$

This paper has been typeset from a $\mathrm{T}_{\mathrm{E}} \mathrm{X} / \mathrm{LAT}_{\mathrm{E}} \mathrm{X}$ file prepared by the author. 